\begin{tabular}{|c|c|}
\hline CRITICAL $\underset{\substack{\text { ECOSYSTEM } \\
\text { PARTERSHIP FUND }}}{\text { ECONS }}$ & $\begin{array}{l}\text { Western Ghats } \\
\text { Special Series }\end{array}$ \\
\hline
\end{tabular}

\section{Chasmophytic grasses of Velliangiri Hills in the southern Western Ghats of Tamil Nadu, India}

\section{Binu Thomas ${ }^{1}$, A. Rajendran ${ }^{2}$, K. Althaf Ahamed Kabeer ${ }^{3} \&$ R. Sivalingam ${ }^{4}$}

1,2,4 Department of Botany, School of Life Sciences, Bharathiar University, Coimbatore, Tamil Nadu 641046, India

${ }^{3}$ Botanical Survey of India, Southern Circle, Coimbatore, Tamil Nadu 641003, India

Email: ${ }^{1}$ binuthomasct@gmail.com, ${ }^{2}$ drarajendra@gmail.com, ${ }^{3}$ althafgrass@gmail.com, ${ }^{4}$ drsivar@gmail.com (corresponding author)

Rock crevices play a key role in forming a major habitat for many plants, and host rich biodiversity within a small area. The rocky habitat provides extremely harsh physical environment for plants that leads to the development of specialized plant communities with endemic and habitat specific species. The microhabitat like rock crevices possess diverse forms of plants, which are mainly seasonal herbs. These habitats differ from each other due to changes in geographical terrain and soil cover (Porembski 2000).

Chasmophytes are plants rooted in clefts of rocks that are filled with detritus. In these clefts particles of

Date of publication (online): 26 December 2012

Date of publication (print): 26 December 2012

ISSN 0974-7907 (online) | 0974-7893 (print)

Editor: N.P. Balakrishnan

Manuscript details:

Ms \# 03107

Received 21 February 2012

Final received 14 October 2012

Finally accepted 29 October 2012

Citation: Thomas, B., A. Rajendran, K.A.A. Kabeer \& R. Sivalingam (2012) Chasmophytic grasses of Velliangiri Hills in the southern Western Ghats of Tamil Nadu, India. Journal of Threatened Taxa 4(15): 3462-3472.

Copyright: ( ) Binu Thomas, A. Rajendran, K. Althaf Ahamed Kabeer \& R Sivalingam 2012. Creative Commons Attribution 3.0 Unported License JoTT allows unrestricted use of this article in any medium for non-profit purposes, reproduction and distribution by providing adequate credit to the authors and the source of publication.

Acknowledgements: We are all thankful to Professor and Head, Department of Botany, Bharathiar University, Coimbatore and Head of Office, Botanical Survey of India, Southern Circle, Coimbatore for providing necessary facilities to carry out the present research.

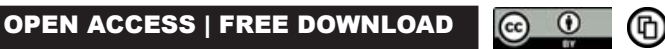

earth conveyed by wind and water accumulate. The amount and rate of accumulationdependupon the width and situation of the clefts (Davis

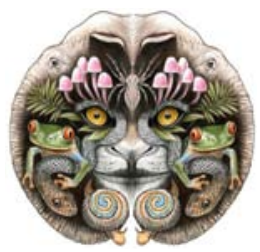
1982). The soil thus constituted facilitates plants to establish and their dead fragments further add to the supply of the nutritive material in the clefts (Bashan et al. 2002). The chasmophytic vegetation inhabiting rock crevices and cliffs represent specific habitat with extreme ecological conditions such as extreme drought, temperature fluctuations, width of the cliffs, rate of accumulation, limited soil volume and scarce nutrients, nature of the rock types, rock hardness and sediment porosity and water holding capacity of the substratum (Nagy \& Proctor 1997; Bashan et al. 2002, 2006).

The grass family occupies $23 \%$ of the land area of the world, playing a significant role in the life of human beings and animals, and has a paramount role as a food provider, accounting for more than $80 \%$ of the world's calories (Kabeer \& Nair 2009). A comprehensive account of the grasses of Tamil Nadu was published by Kabeer \& Nair (2009) in their floristic studies. However, there has been no study of chasmophytic features of grasses as yet. A comprehensive study was carried out to assess the chasmophytic diversity of grasses from Velliangiri Hills of southern Western Ghats of Tamil Nadu (Fig. 1).

Study area and Methods: Velliangiri Hills are floristically rich and socio-religiously important range of southern Western Ghats situated 40km west of Coimbatore City, Tamil Nadu. The study area lies between $6^{0} 40^{\prime}-7^{0} 10^{\prime} \mathrm{E}$ and $10^{\circ} 55^{\prime}-11^{\circ} 10^{\prime} \mathrm{N}$ between $520-1840 \mathrm{~m}$. A famous temple called "Velliangiri Aandavar' temple also called "Thenkailayam" (South Kailas) is situated at the peak of the hills $(1840 \mathrm{~m})$. The range of study area consists of seven hills with different altitudes and topography.

Correct nomenclature, habit, habitat, phenology and pattern of distribution available are given (Table 1). Plant specimens were identified with regional and local floras (Gamble \& Fischer 1988; Mathew 1983; Chandrabose \& Nair 1988; Henry et al. 1989; Kabeer \& Nair 2009). The voucher specimens are deposited in the herbarium

The publication of this article is supported by the Critical Ecosystem Partnership Fund (CEPF), a joint initiative of l'Agence Française de Développement, Conservation International, the European Commission, the Global Environment Facility, the Government of Japan, the MacArthur Foundation and the World Bank. 


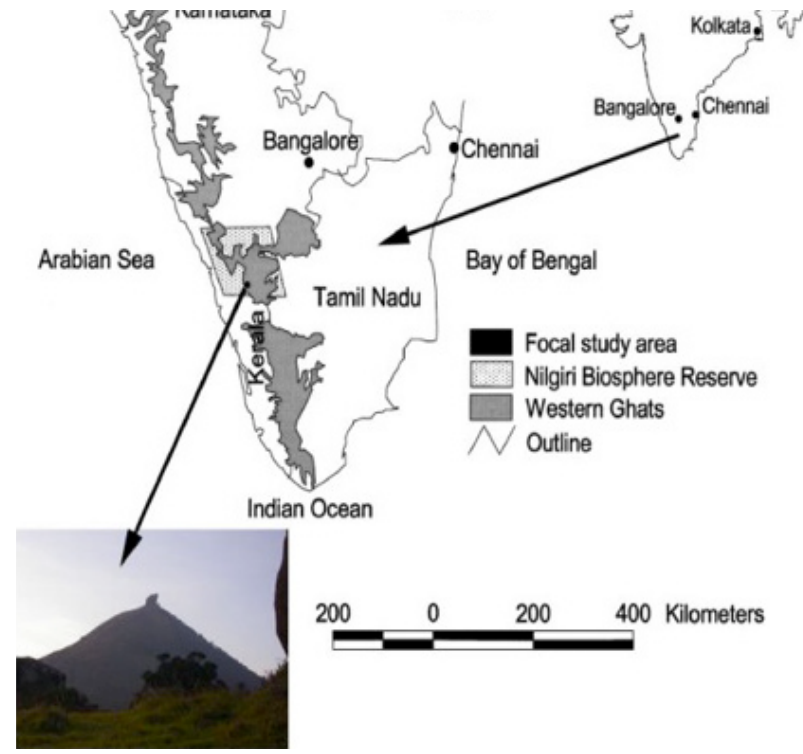

Figure 1. Study area

of Botany Department, Bharathiar University (BUH), Coimbatore, Tamil Nadu, India.

Results and Discussion: The data presented here are the outcome of a series of extensive and intensive studies conducted during September 2010-October 2011 had resulted in the documentation and collection of 30 species of wild chasmophytic grass taxa from Velliangiri Hills of southern Western Ghats of Tamil Nadu (Images $1-35)$.

The present study incorporated 30 species of chasmophytic grasses distributed in 26 genera (Table 1). Among these genera Eragrostis is the dominant genus with four species, namely, aspera, tenella, nigra and uniloides. Some of the notable chasmophytic grasses are used by the local tribe 'Malasars'. The stalks of Apluda mutica are used for making hats. Cymbopogon flexuosus is used to extract the lemon grass oil for medicinal purposes. Ash of Pogonatherum crinitum are used for skin problems. The spikelets of Setaria palmifolia and Melinus repens are highly attractive and used ornamentally. Most of the grasses are used as fodder.

Some of the threats like heavy influence of pilgrims, recreational pressures, collection of fire wood, lack of suitable management and other construction activities adversely affect the existing ecosystem. It is suggested that the chasmophytic vegetation needs to be protected through sustainable utilization.

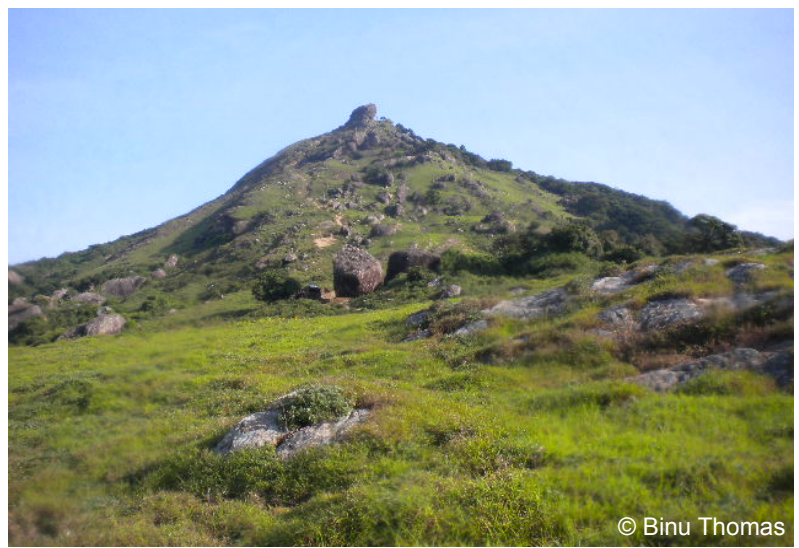

Image 1. View of Velliangiri hill top

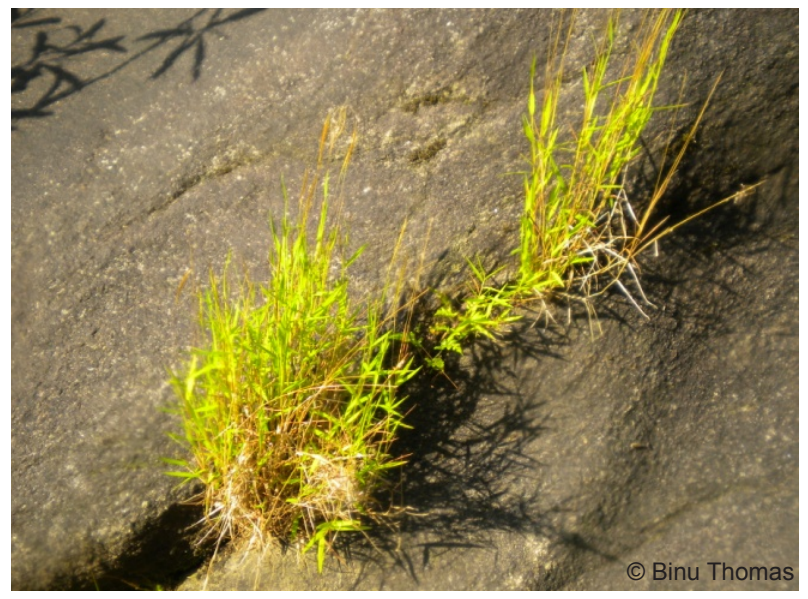

Image 2. Pogonatherum crinitum (Thunb.) Kunth.

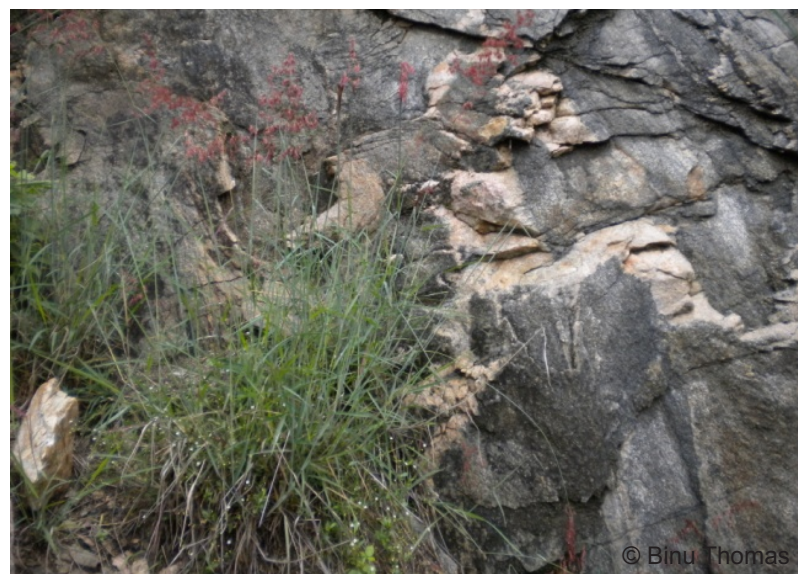

Image 3. Melinis repens (Willd.) Zizka

\section{REFERENCES}

Bashan, Y., H. Vierheilig \& B.G. Salazar (2006). Primary colonization and breakdown of igneous rocks by endemic succulent plants of the deserts in Baja California, Mexico. Naturwissenschaften 93: 344-347. 
Table 1. Chasmophytic grasses of Velliangiri Hills, southern Western Ghats of Tamil Nadu

\begin{tabular}{|c|c|c|c|c|c|}
\hline Sno & Botanical name (accession number) & Habit & Habitat & Phenology & Distribution \\
\hline 1 & Apluda mutica L. (BUH: 7298) (Image 6) & Tufted perennial & $\begin{array}{l}\text { Rock crevices of hill slopes up } \\
\text { to } 1700 \mathrm{~m}\end{array}$ & $\begin{array}{l}\text { Throughout } \\
\text { the year }\end{array}$ & Common \\
\hline 2 & $\begin{array}{l}\text { Arthraxon hispidus (Thumb.) Makin. } \\
\text { (BUH: 7123) (Image 7) }\end{array}$ & Tufted annual & $\begin{array}{l}\text { Rock crevices of hill slopes up to } \\
1800 \mathrm{~m} .\end{array}$ & Apr-Feb & Uncommon \\
\hline 3. & $\begin{array}{l}\text { Arundinella pumila (Hochst. ex A. Rich) Steud. } \\
\text { (BUH: 7126) (Image 8) }\end{array}$ & Tufted annual & On dripping rocks & Nov-Mar & Rare \\
\hline 4. & $\begin{array}{l}\text { Axonopus compressus (Sw.) P. Beauv. } \\
\text { (BUH: 7125) (Image 9) }\end{array}$ & Perennial grass & Rock crevices up to $1500 \mathrm{~m}$ & Jun-Feb & Common \\
\hline 5. & $\begin{array}{l}\text { Capillipedium assimile (Steud.) A. Camus } \\
\text { (BUH: 7142) (Image 10) }\end{array}$ & Tufted perennial & Cliffs of hill slopes $900-1800 \mathrm{~m}$ & Oct-Mar & Uncommon \\
\hline 6. & Cenchrus ciliaris L. (BUH: 7151) (Image 11) & $\begin{array}{l}\text { Stoloniferous } \\
\text { perennial }\end{array}$ & Moist places of rocky cliffs & $\begin{array}{l}\text { Throughout } \\
\text { the year }\end{array}$ & Common \\
\hline 7. & $\begin{array}{l}\text { Centotheca lappacea (L.) Desv. } \\
\text { (BUH: 7153) (Image 12) }\end{array}$ & $\begin{array}{l}\text { Decumbent } \\
\text { perennial }\end{array}$ & Moist shaded places of hilly cliffs & Apr-Nov & Uncommon \\
\hline 8. & $\begin{array}{l}\text { Cryptococcum oxyphyllum (Steud.) Stapf. } \\
\text { (BUH: } 7174 \text { ) (Image 13) }\end{array}$ & Tufted perennial & $\begin{array}{l}\text { Rock crevices of forest floor up } \\
\text { to } 1400 \mathrm{~m}\end{array}$ & Jul-Apr & Uncommon \\
\hline 9. & $\begin{array}{l}\text { Cymbopogon flexuosus (Nees ex Steud.) Will. } \\
\text { (BUH: 7183) (Image 14) }\end{array}$ & Tufted perennial & Grassland rocky cliffs at $1700 \mathrm{~m}$ & Jul-Apr & Common \\
\hline 10. & $\begin{array}{l}\text { Cryptococcum trigonum (Retz.) A. Camus } \\
\text { (BUH: 7389) (Image 15) }\end{array}$ & $\begin{array}{l}\text { Stoloniferous } \\
\text { perennial }\end{array}$ & Cliffs of moist shady places & Jul-Apr & Uncommon \\
\hline 11. & $\begin{array}{l}\text { Eleusine indica (L.) Gaertn. } \\
\text { (BUH: 7297) (Image 16) }\end{array}$ & Tufted annual & Rocky cliffs at $600-800 \mathrm{~m}$ & $\begin{array}{l}\text { Throughout } \\
\text { the year }\end{array}$ & Uncommon \\
\hline 12. & $\begin{array}{l}\text { Eragrostis aspera (Jacq.) Nees. } \\
\text { (BUH: } 7200 \text { ) (Image 17) }\end{array}$ & Annual & Rocky cliffs of hill slopes & Nov-Feb & Rare \\
\hline 13. & $\begin{array}{l}\text { Eragrostis nigra Nees ex Steud. } \\
\text { (BUH: 7203) (Image 18) }\end{array}$ & Tufted perennial & Rock crevices of marshy areas & June-Mar & Uncommon \\
\hline 14. & $\begin{array}{l}\text { Eragrostis tenella (L.) P. Beauv. } \\
\text { (BUH: 7202) (Image 19) }\end{array}$ & Tufted annual & Rocky cliffs of foot hills & $\begin{array}{l}\text { Throughout } \\
\text { the year }\end{array}$ & Uncommon \\
\hline 15. & $\begin{array}{l}\text { Eragrostis uniloides (Retz.) Nees ex Steud. } \\
\text { (BUH: 7204) (Image 20) }\end{array}$ & Tufted annual & Rock crevices of marshy areas & $\begin{array}{l}\text { Throughout } \\
\text { the year }\end{array}$ & Uncommon \\
\hline 16. & $\begin{array}{l}\text { Garnotia arundinacea Hook. } \\
\text { (BUH: 7202) (Image 21) }\end{array}$ & Tufted annual & $\begin{array}{l}\text { Rock crevices of open areas at } \\
\text { about } 1500 \mathrm{~m}\end{array}$ & Jul-Feb & Uncommon \\
\hline 17. & $\begin{array}{l}\text { Heteropogon contortus (L.) P. Beauv. } \\
\text { (BUH: 7220) (Image 22) }\end{array}$ & Tufted perennial & $\begin{array}{l}\text { Rock crevices of hills at about } \\
400-600 \mathrm{~m}\end{array}$ & $\begin{array}{l}\text { Throughout } \\
\text { the year }\end{array}$ & Common \\
\hline 18. & $\begin{array}{l}\text { Melinis repens (Willd.) Zizka } \\
\text { (BUH: 7267) (Image 23) }\end{array}$ & Tufted annual & $\begin{array}{l}\text { Rocky cliffs of open dry areas at } \\
\text { about } 600-800 \mathrm{~m}\end{array}$ & $\begin{array}{l}\text { Throughout } \\
\text { the year }\end{array}$ & Common \\
\hline 19. & $\begin{array}{l}\text { Oplismenus compositus (L.) P. Beauv. } \\
\text { (BUH: 7275) (Image 24) }\end{array}$ & Creeping annual & Rocky cliffs of shaded areas & Jul-Mar & Common \\
\hline 20. & $\begin{array}{l}\text { Panicum curviflorum Hornem. (Samaikarunai) } \\
\text { (BUH: 7281) (Image 25) }\end{array}$ & Tufted annual & $\begin{array}{l}\text { Rocky cliffs of grass lands at } \\
\text { about } 1300 \mathrm{~m}\end{array}$ & May-Feb & Common \\
\hline 21. & $\begin{array}{l}\text { Paspalidium flavidum (Retz.) A. Camus } \\
\text { (BUH: 7282) (Image 26) }\end{array}$ & Tufted annual & Moist shady places of rocky cliffs & May-Mar & Uncommon \\
\hline 22. & $\begin{array}{l}\text { Pennisetum polystachion (L.) Schult. } \\
\text { (BUH: 7286) (Image 27) }\end{array}$ & Tufted annual & $\begin{array}{l}\text { Rocky cliffs of marshy areas at } \\
1250 \mathrm{~m}\end{array}$ & Jul-Apr & Uncommon \\
\hline 23. & $\begin{array}{l}\text { Pogonatherum crinitum (Thunb.) Kunth. (BUH: } \\
\text { 7310) (Image 28) }\end{array}$ & Tufted annual & $\begin{array}{l}\text { Rocky cliffs of hill slopes at } \\
1200 \mathrm{~m}\end{array}$ & May-Mar & Common \\
\hline 24. & $\begin{array}{l}\text { Rottboellia cochinchinensis (Lour.) Clayton } \\
\text { (BUH: 7325) (Image 29) }\end{array}$ & Tufted perennial & $\begin{array}{l}\text { Rocky cliffs along streams at } \\
1200 \mathrm{~m}\end{array}$ & $\begin{array}{l}\text { Throughout } \\
\text { the year }\end{array}$ & Uncommon \\
\hline 25. & $\begin{array}{l}\text { Setaria palmifolia (J. Koenig) Stapf. } \\
\text { (BUH: 7333) (Image 30) }\end{array}$ & Tufted perennial & Rocky cliffs of marshy areas & Jul-Apr & Uncommon \\
\hline 26. & $\begin{array}{l}\text { Sorghum halepense (L.) Pers. } \\
\text { (BUH: 7337) (Image 31) }\end{array}$ & $\begin{array}{l}\text { Rhizomatous } \\
\text { perennial }\end{array}$ & $\begin{array}{l}\text { Rock crevices of hills at about } \\
600-800 \mathrm{~m}\end{array}$ & Oct-Jan & Common \\
\hline 27. & $\begin{array}{l}\text { Spodiopogon rhizophorus (Steud.) Pilger } \\
\text { (BUH: 7202) (Image 32) }\end{array}$ & Tufted annual & $\begin{array}{l}\text { Rocky cliffs along hill slopes at } \\
1300 \mathrm{~m}\end{array}$ & Nov-Dec & Uncommon \\
\hline 28. & $\begin{array}{l}\text { Sporobolus indicus (L.) R. Br. var. flaccidus } \\
\text { (Roem \& Schult) (BUH: 7341) (Image 33) }\end{array}$ & Tufted annual & $\begin{array}{l}\text { Rocky cliffs of forest floor at } \\
600-800 \mathrm{~m}\end{array}$ & May-Mar & Common \\
\hline 29. & $\begin{array}{l}\text { Themeda triandra Forssk. (Erigaithattuppullu) } \\
\text { (BUH: 7341) (Image 34) }\end{array}$ & Tufted perennial & Rocky cliffs of hill slopes & May-Mar & Common \\
\hline 30. & $\begin{array}{l}\text { Zenkeria elegans Trin. (Kallubothai) } \\
\text { (BUH: 7362) (Image 35) }\end{array}$ & $\begin{array}{l}\text { Tufted rhizomatous } \\
\text { perennial }\end{array}$ & $\begin{array}{l}\text { Massive clumps in rocky areas } \\
\text { at } 1700 \mathrm{~m}\end{array}$ & Jun-Jan & Uncommon \\
\hline
\end{tabular}




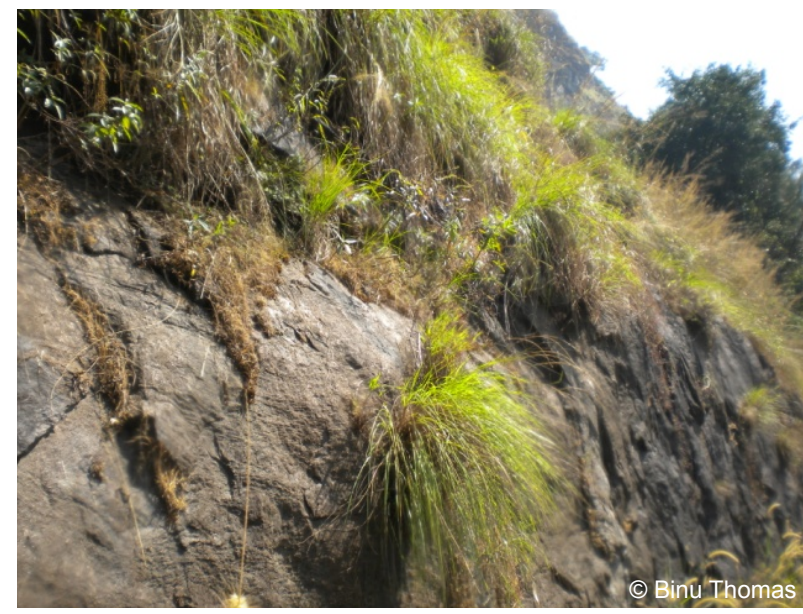

Image 4. Sporobolus indicus (L.) R. Br. var. flaccidus

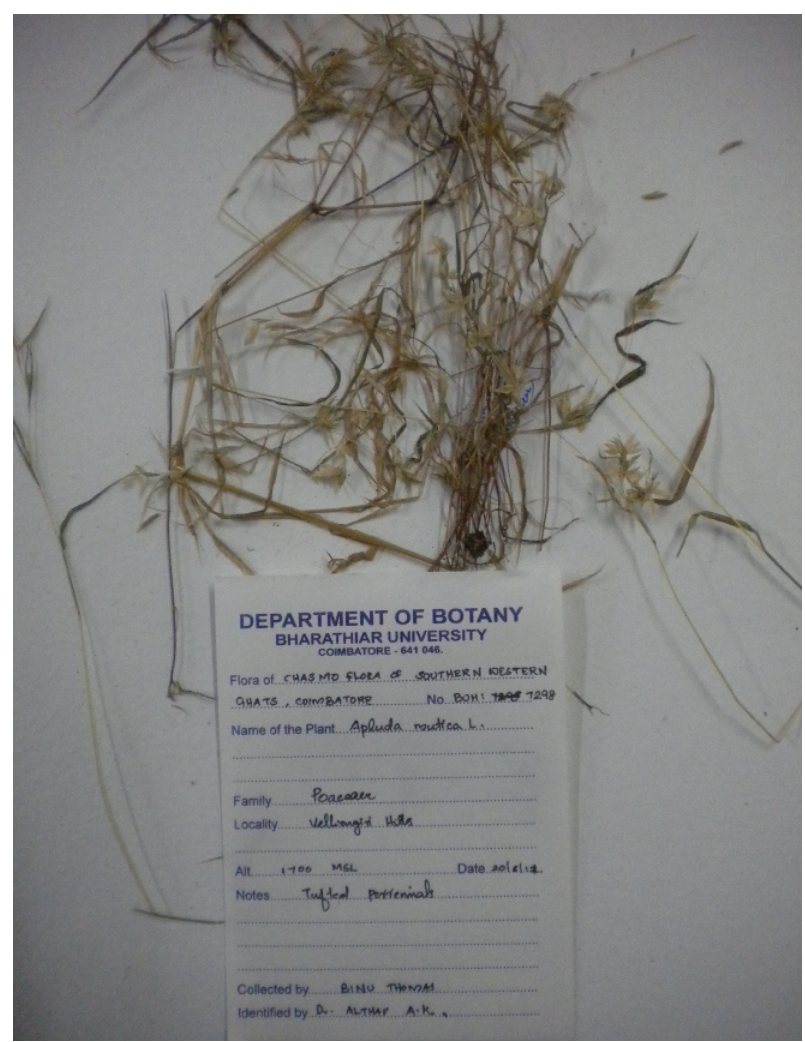

Image 6. Apluda mutica L.

Bashan, Y., C.Y. Li., V.K. Lebsky \& M. Monero (2002). Colonization of chasmophytic plants in arid Baja California, Mexico. Plant Biology 4: 392-402.

Chandrabose, M. \& N.C. Nair (1988). Flora of Coimbatore. Botanical Survey of India, Coimbatore, 322-356pp.

Davis, P.H. (1982). Cliff vegetation in the Eastern Mediterranean. Journal of Ecology 39: 63-93.

Henry, A.N., V. Chitra \& N.P. Balakrishnan (1989). Flora of Tamil Nadu. Analysis-Volume 3. Botanical Survey of India, 87-146pp.

Kabeer, K.A.A. \& V.J. Nair (2009). Flora of Tamil Nadu -

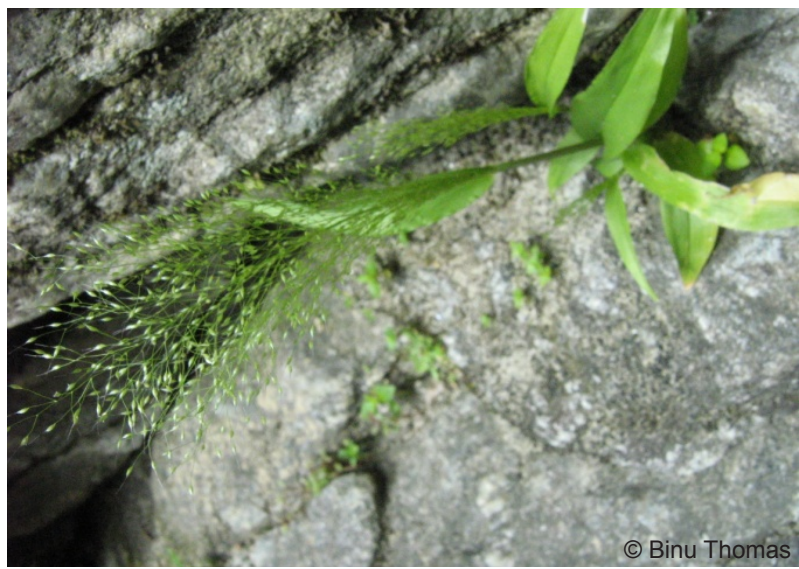

Image 5. Arundinella pumila (Hochst. ex A. Rich.) Steud.

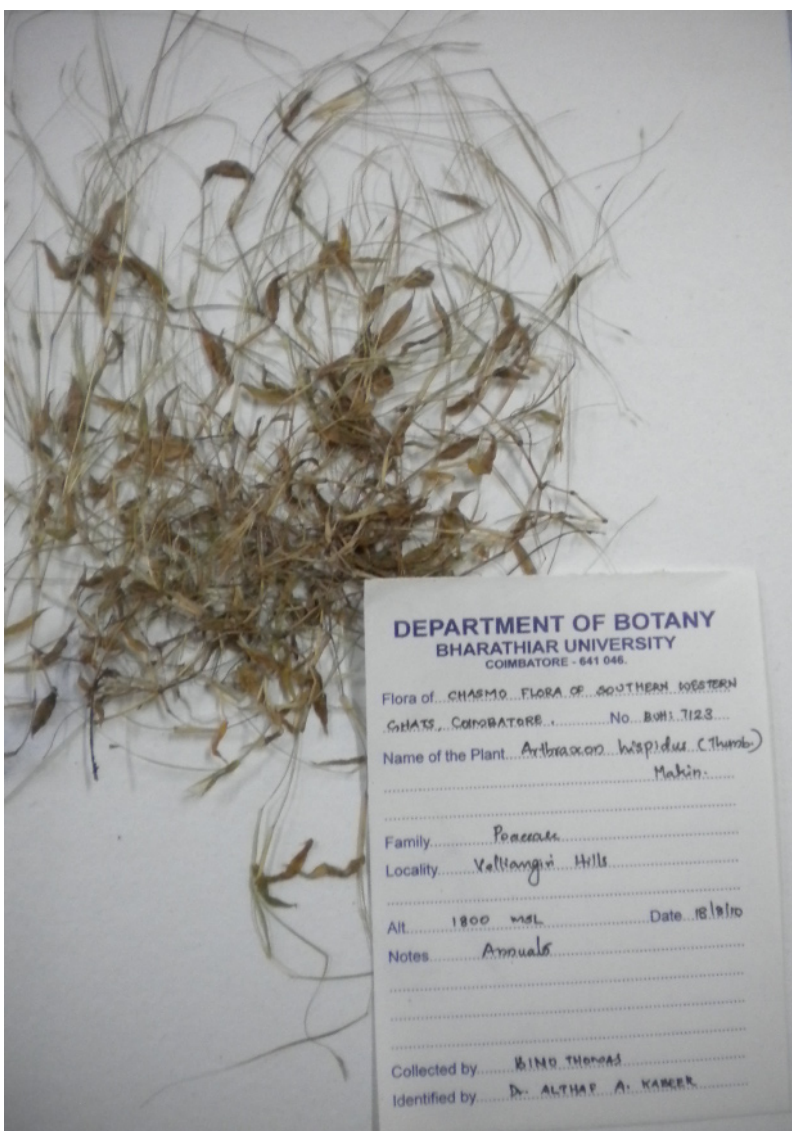

Image 7. Arthraxon hispidus (Thunb.) Makin.

Grasses. Botanical Survey of India, Calcutta, 1-525pp.

Mathew, K.M. (1983). The Flora of The Tamilnadu CarnaticVolumes 1-3. The Rapinat Herbarium, St. Joseph's college, Thiruchirapalli, 1789-1915pp.

Nagy, L. \& J. Proctor (1997). Soil Mg and Ni as casual factors of plant occurrence and distribution at the Meikle Kilrannoch Ultramafic site in Scotland. New Phytology 135: 561-566.

Porembski, S. (2000). Biotic diversity of isolated rock outcrops in tropical and temperate regions. Journal of Ecology 146: 177-208. 


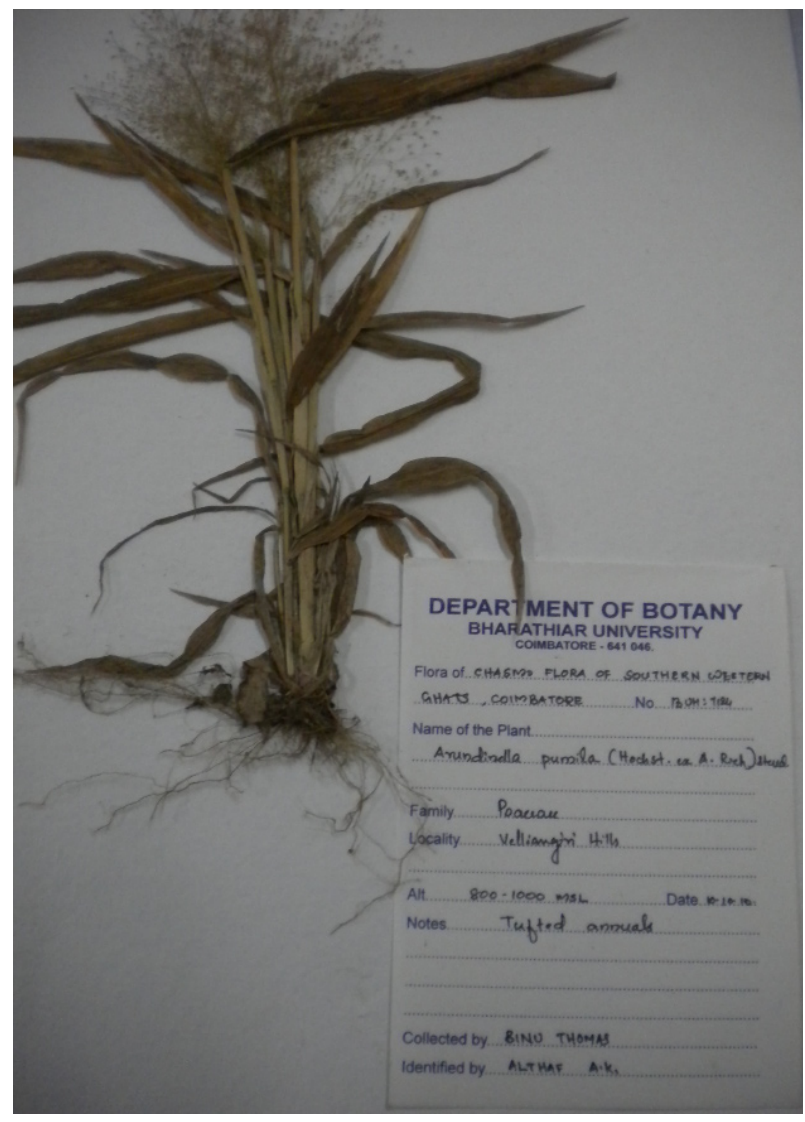

Image 8. Arundinella pumila (Hochst. ex A. Rich.) Steud.

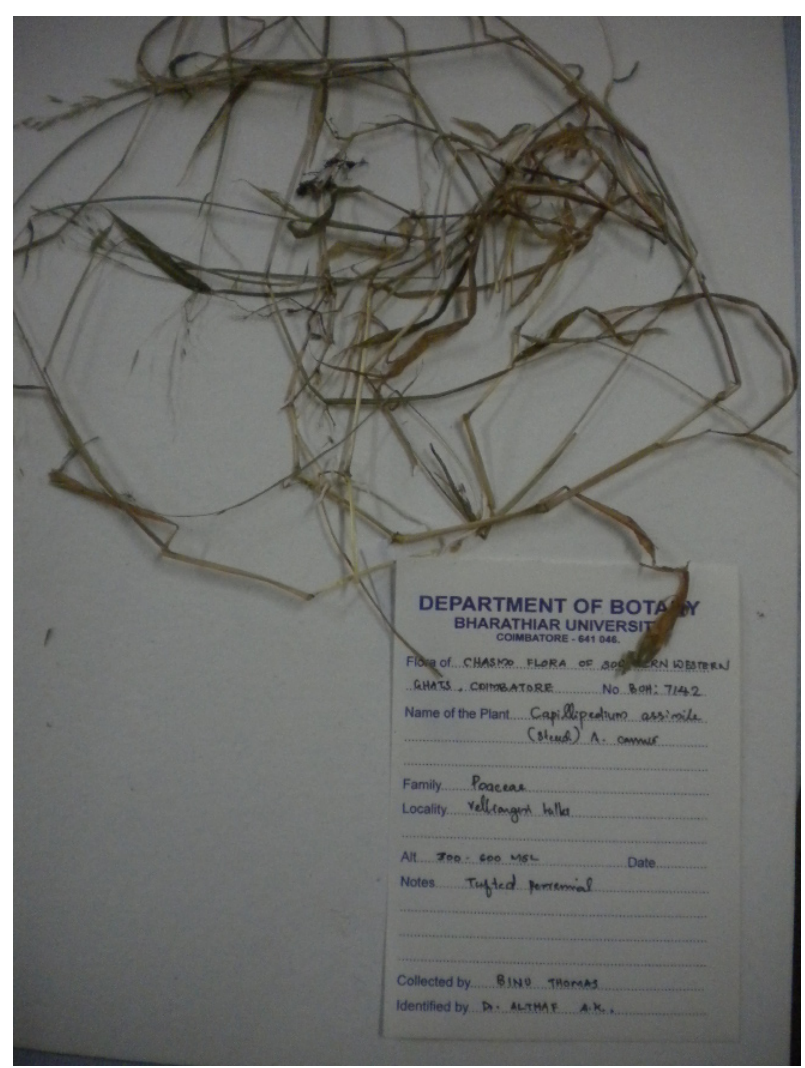

Image 10. Capillipedium assimile (Steud.) A. Camus

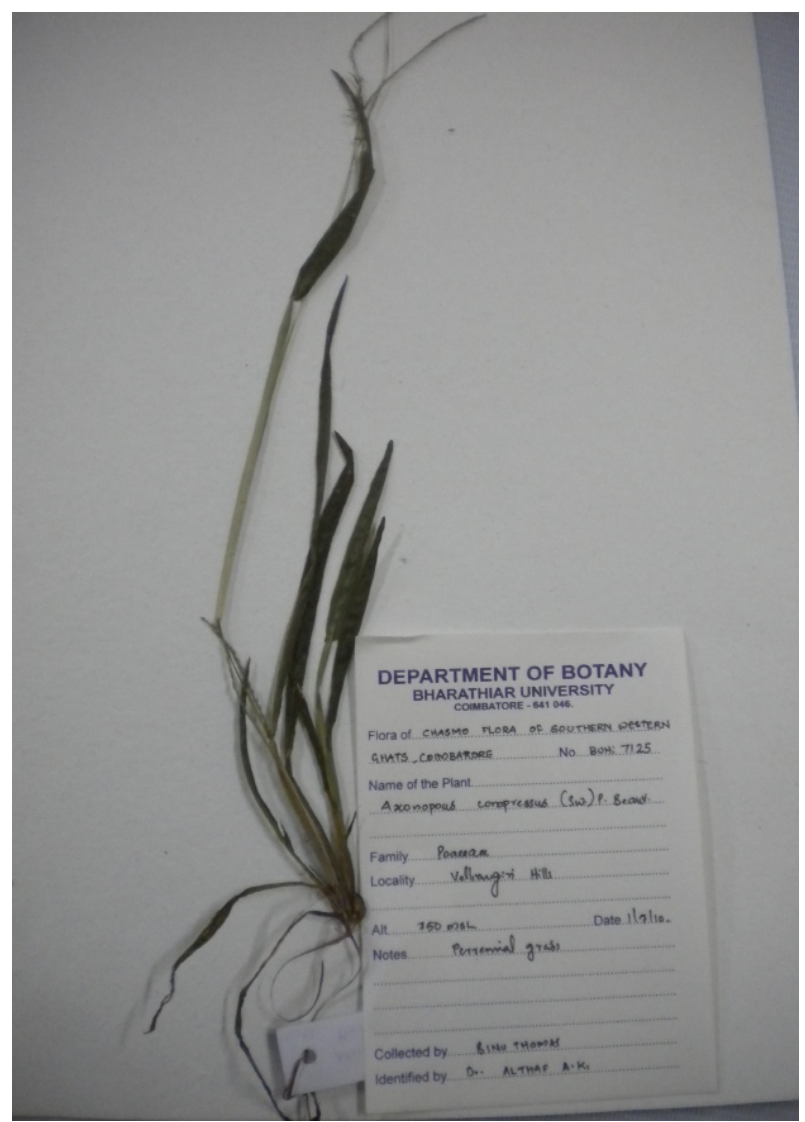

Image 9. Axonopus compressus (Sw.) P. Beauv.

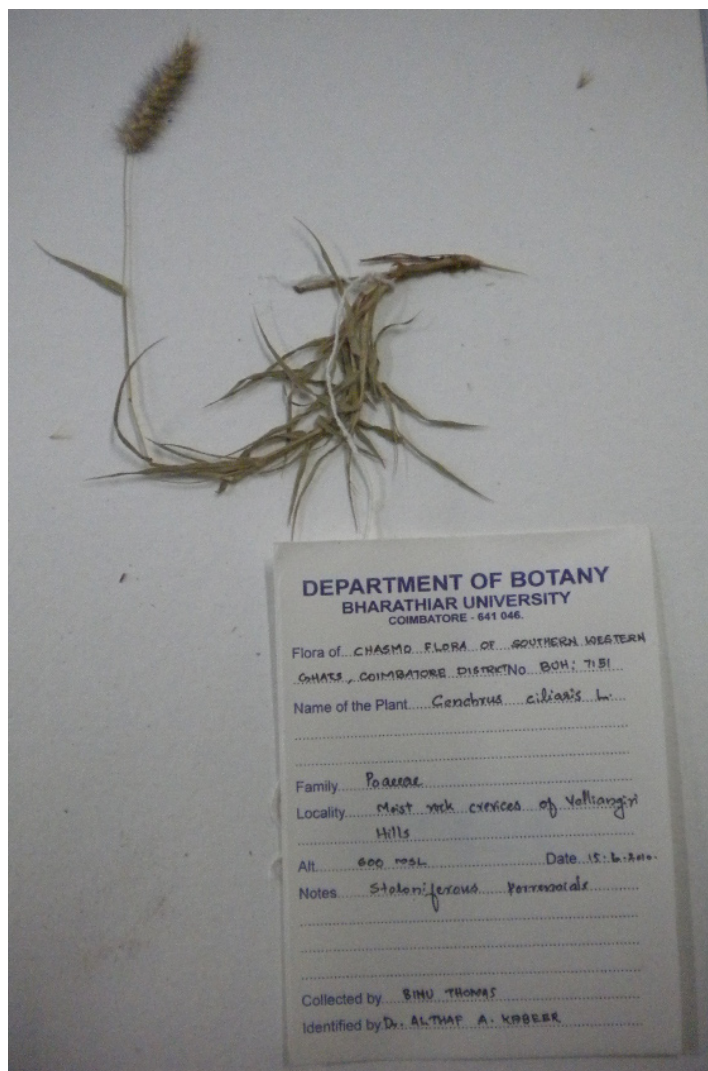

Image 11. Cenchrus ciliaris L. 


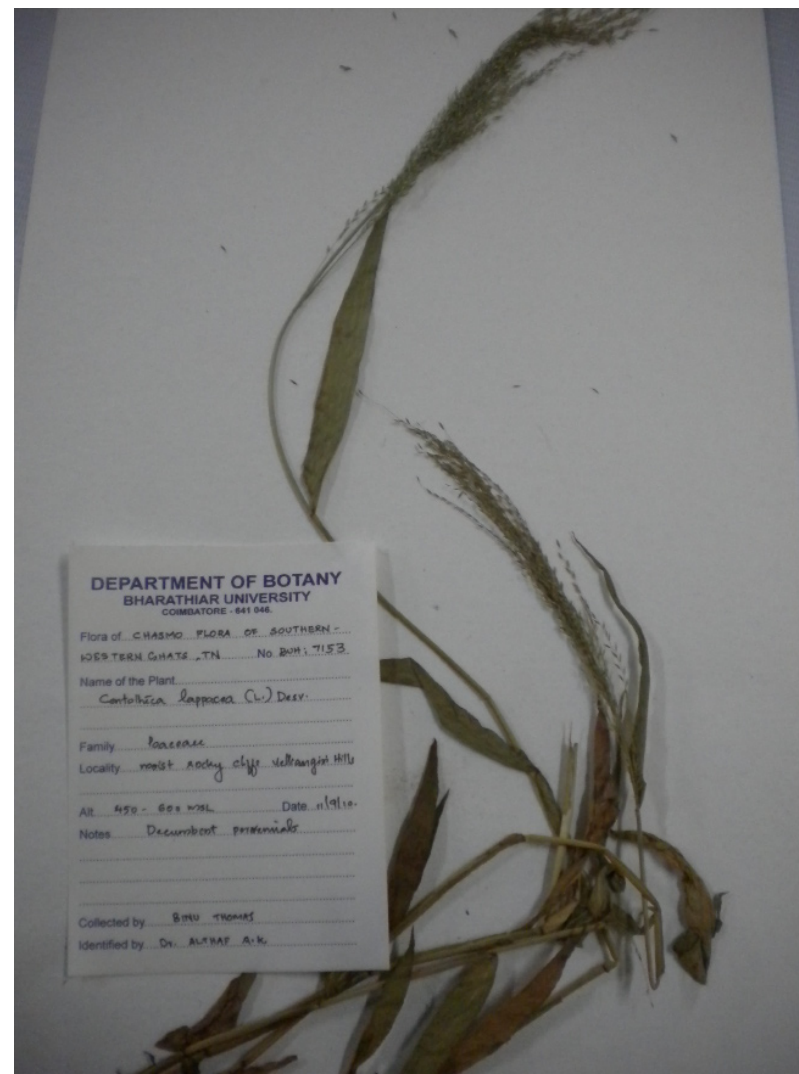

Image 12. Centotheca lappacea (L.) Desv.

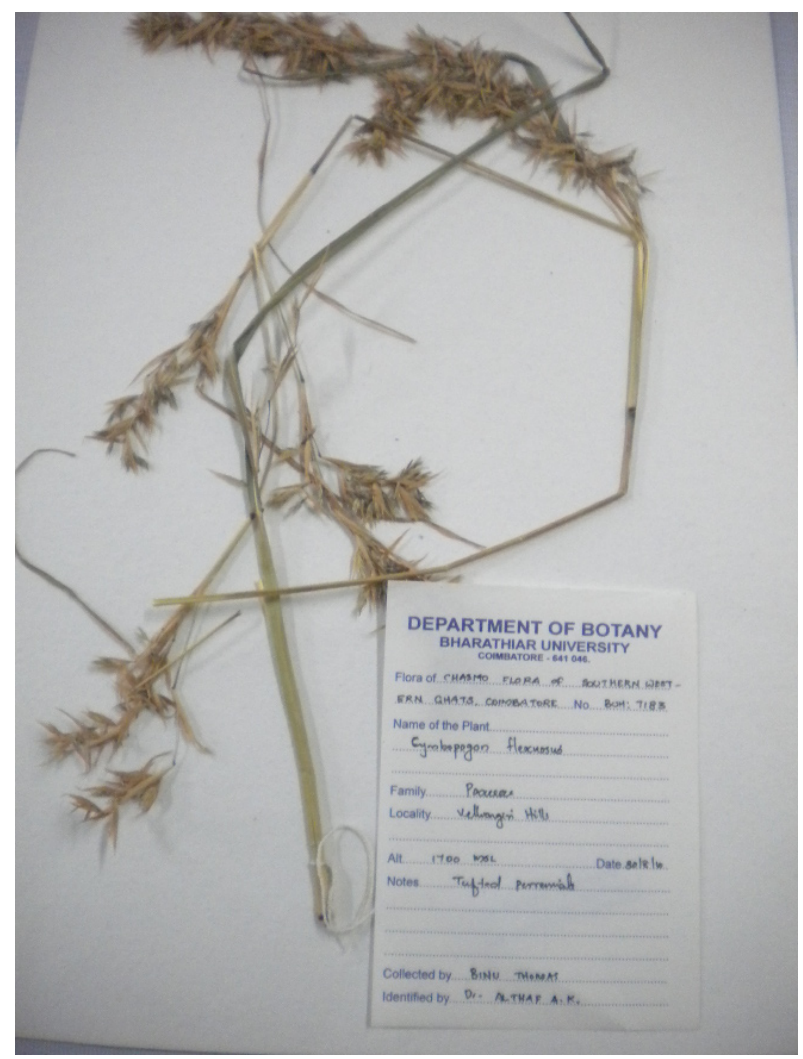

Image 14. Cymbopogon flexuosus (Nees ex Steud.) Will.

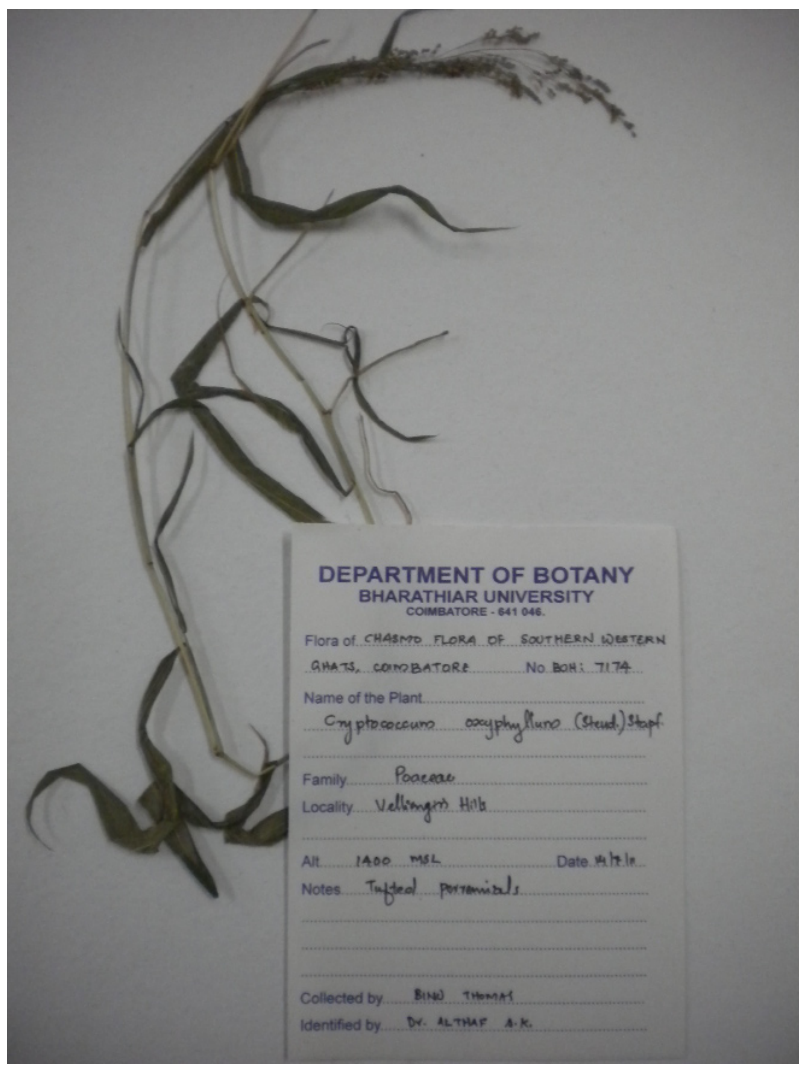

Image 13. Cryptococcum oxyphyllum (Steud.) Stapf

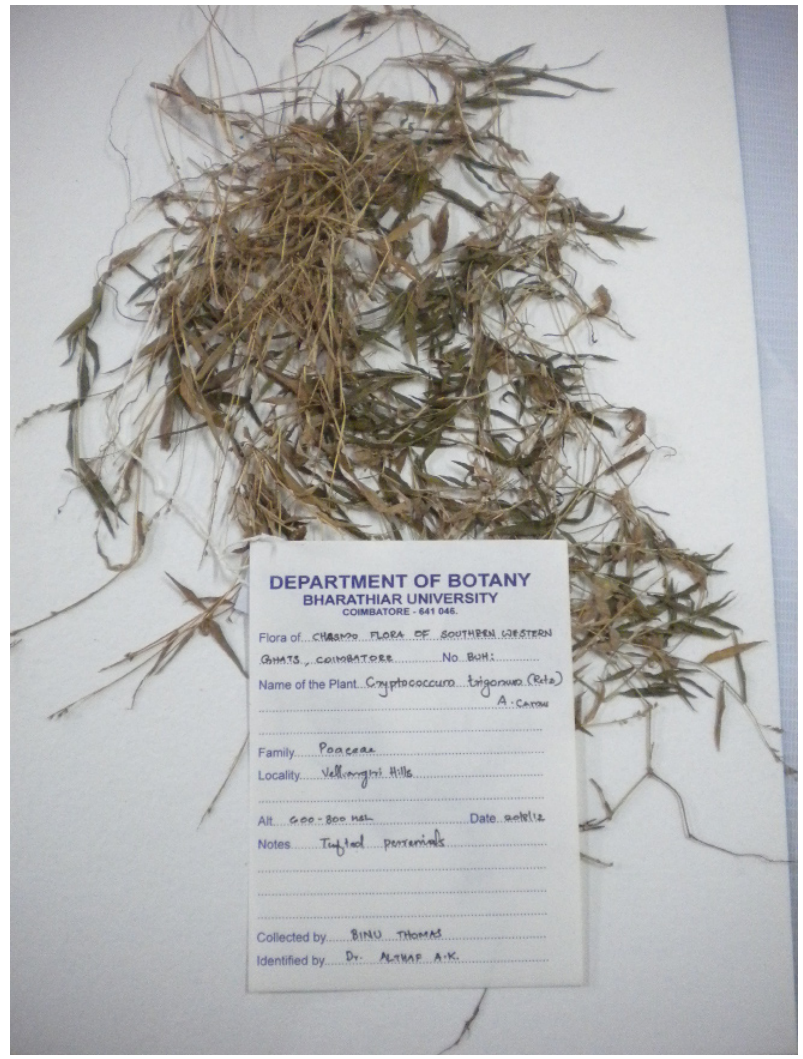

Image 15. Cryptococcum trigonum (Retz.) A. Camus 


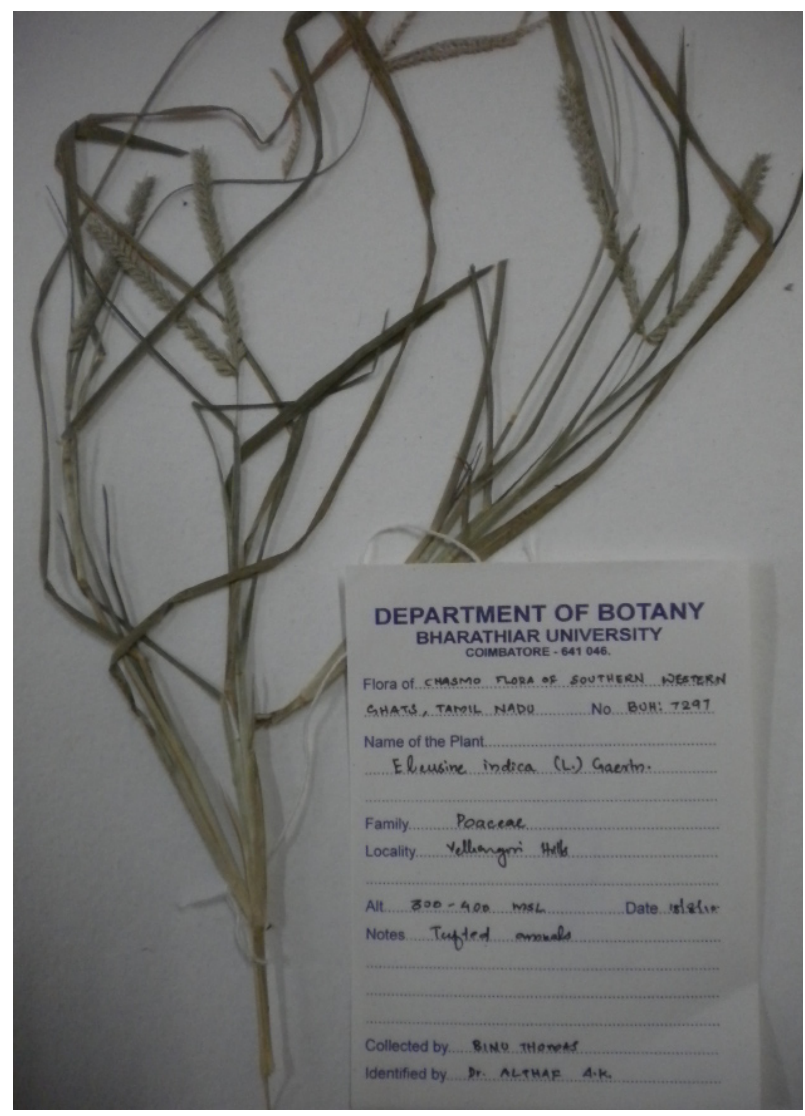

Image 16. Eleusine indica (L.) Gaertn.

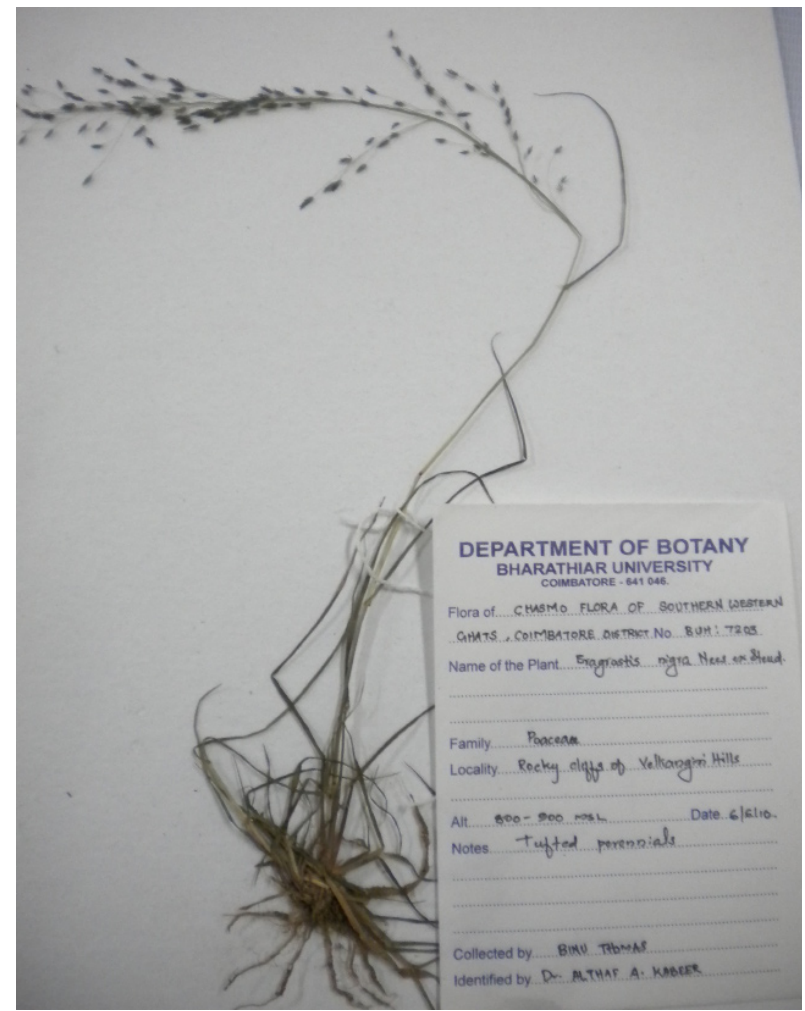

Image 18. Eragrostis nigra Nees ex Steud.

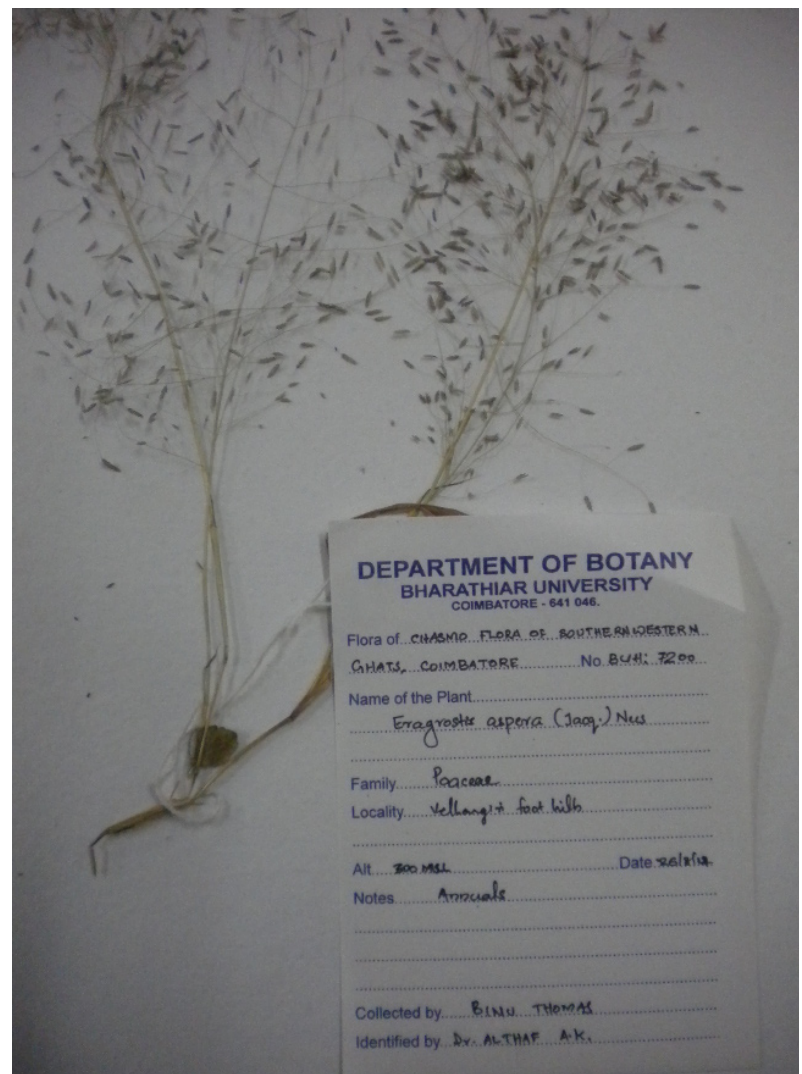

Image 17. Eragrostis aspera (Jacq.) Nees

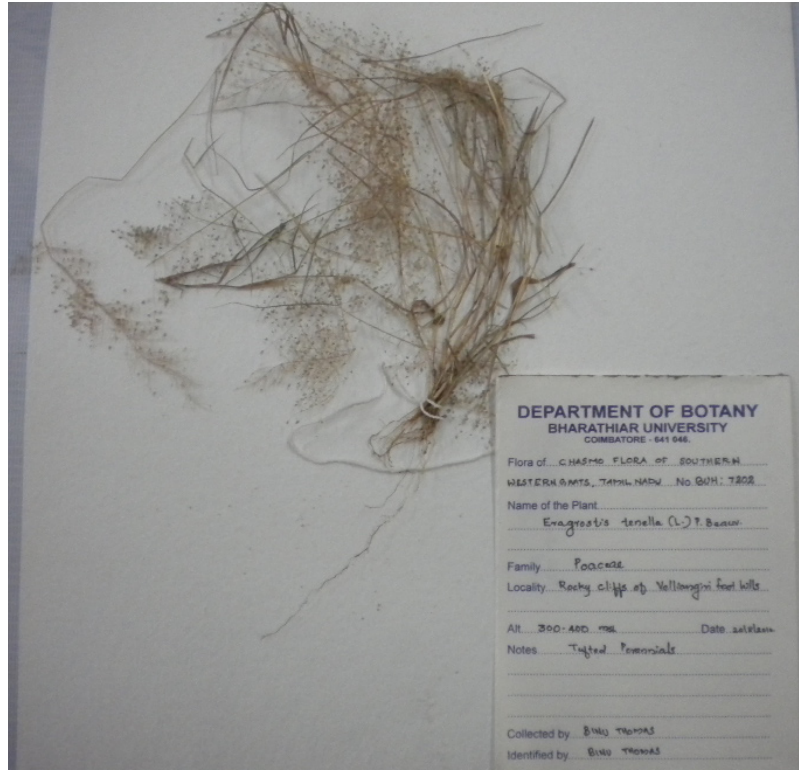

Image 19. Eragrostis tenella (L.) P. Beauv. 


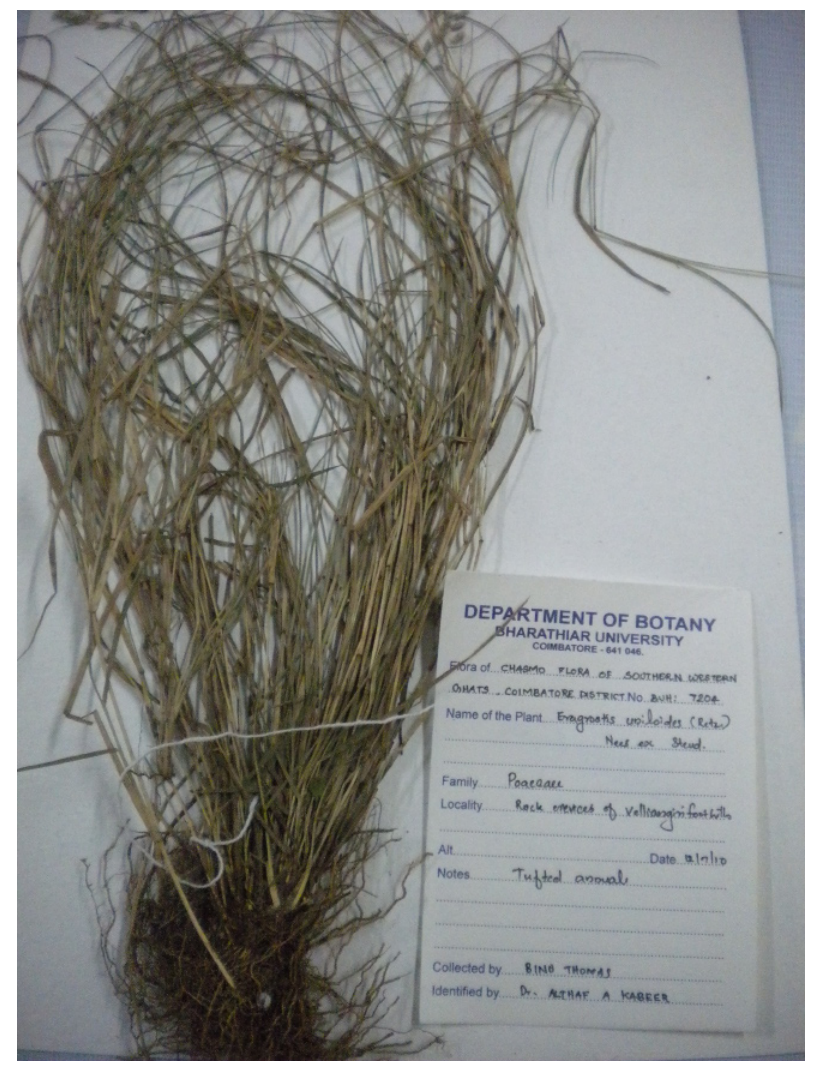

Image 20. Eragrostis uniloides (Retz.) Nees ex Steud.

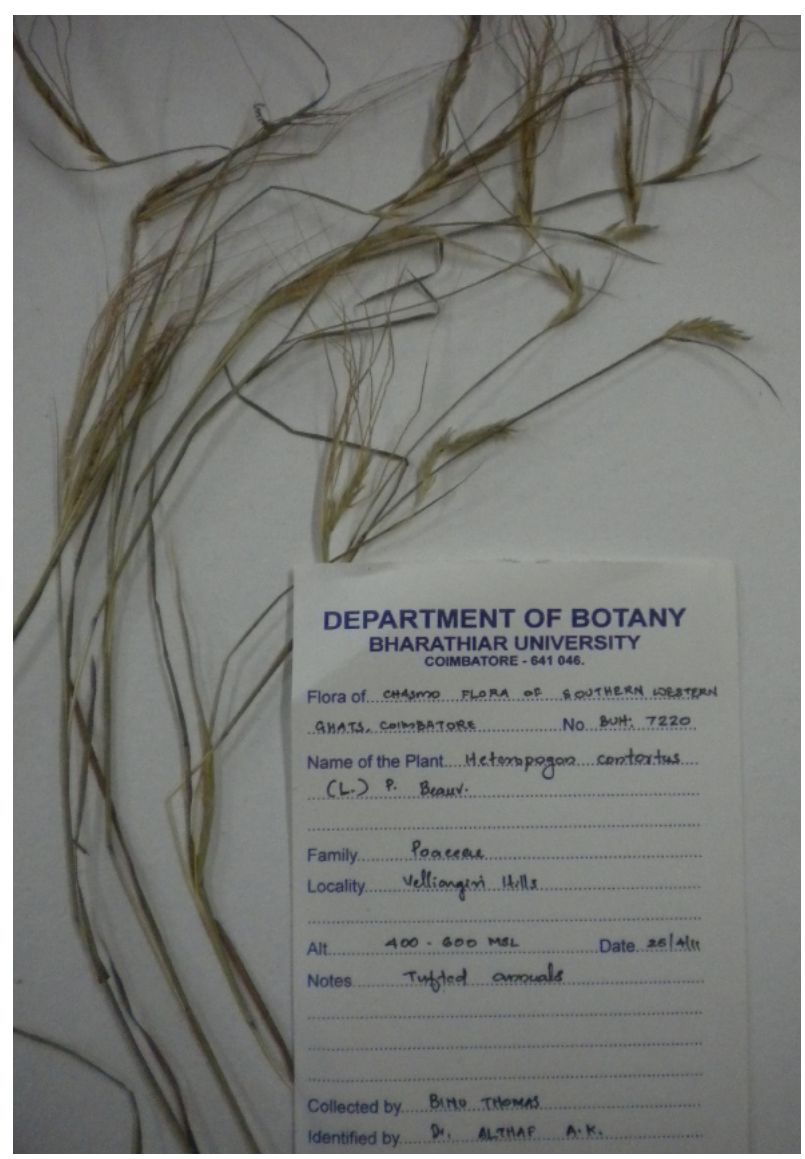

Image 22. Heteropogon contortus (L.) P. Beauv.

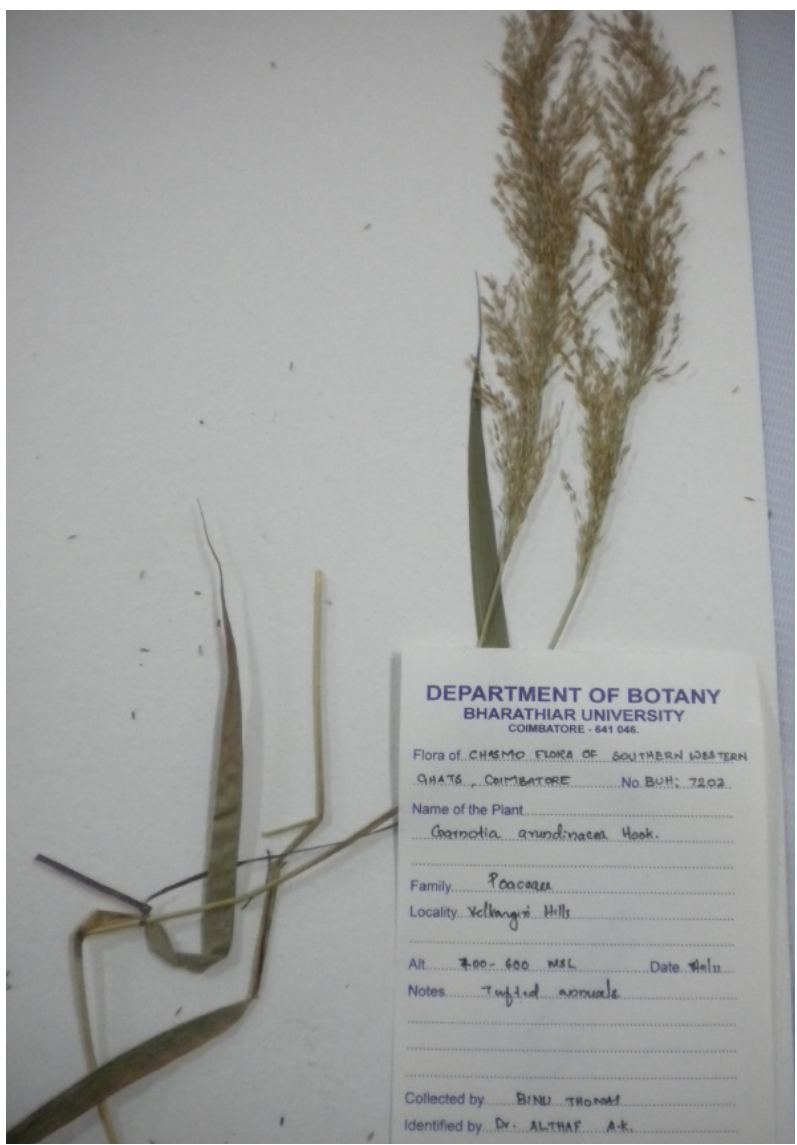

Image 21. Garnotia arundinacea Hook.

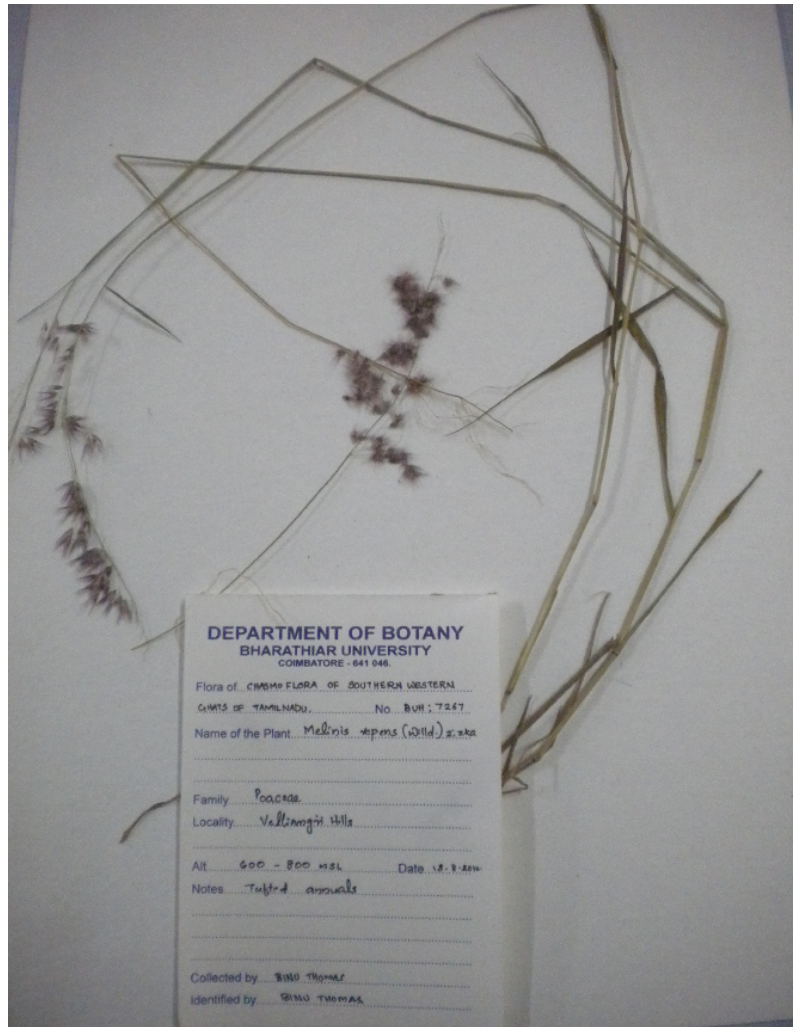

Image 23. Melinis repens (Willd.) Zizka

Journal of Threatened Taxa | www.threatenedtaxa.org | December 2012 | 4(15): 3462-3472 


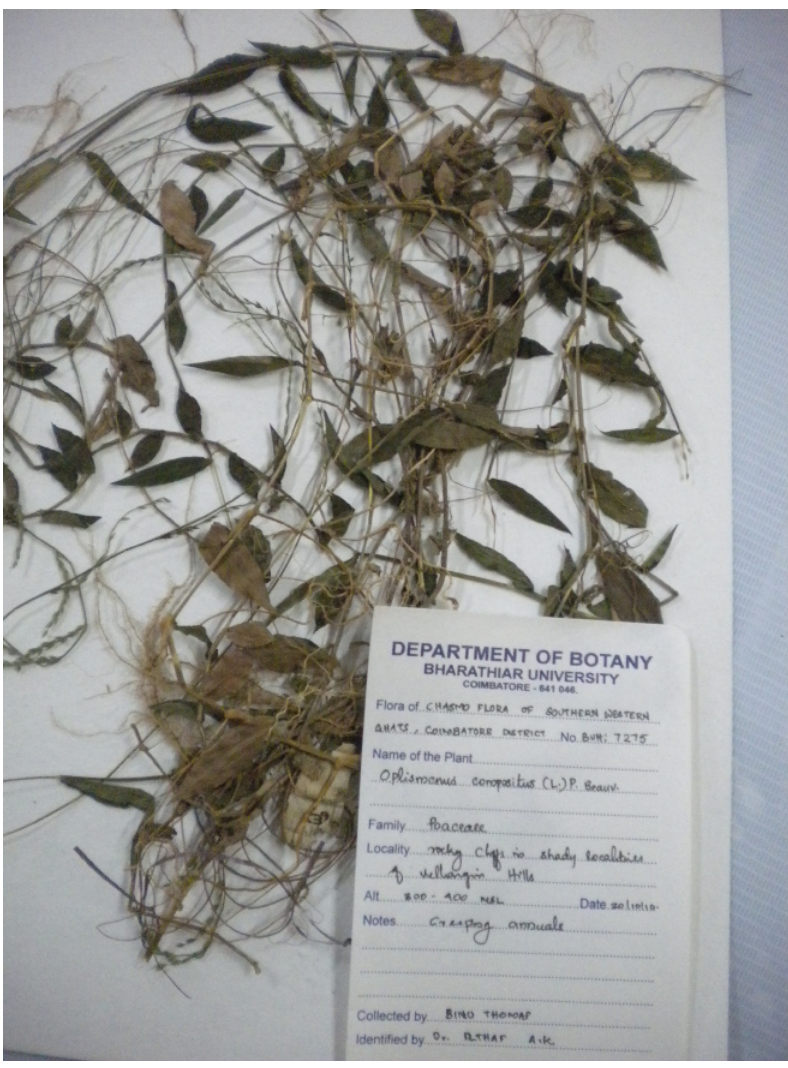

Image 24. Oplismenus compositus (L.) P. Beauv.

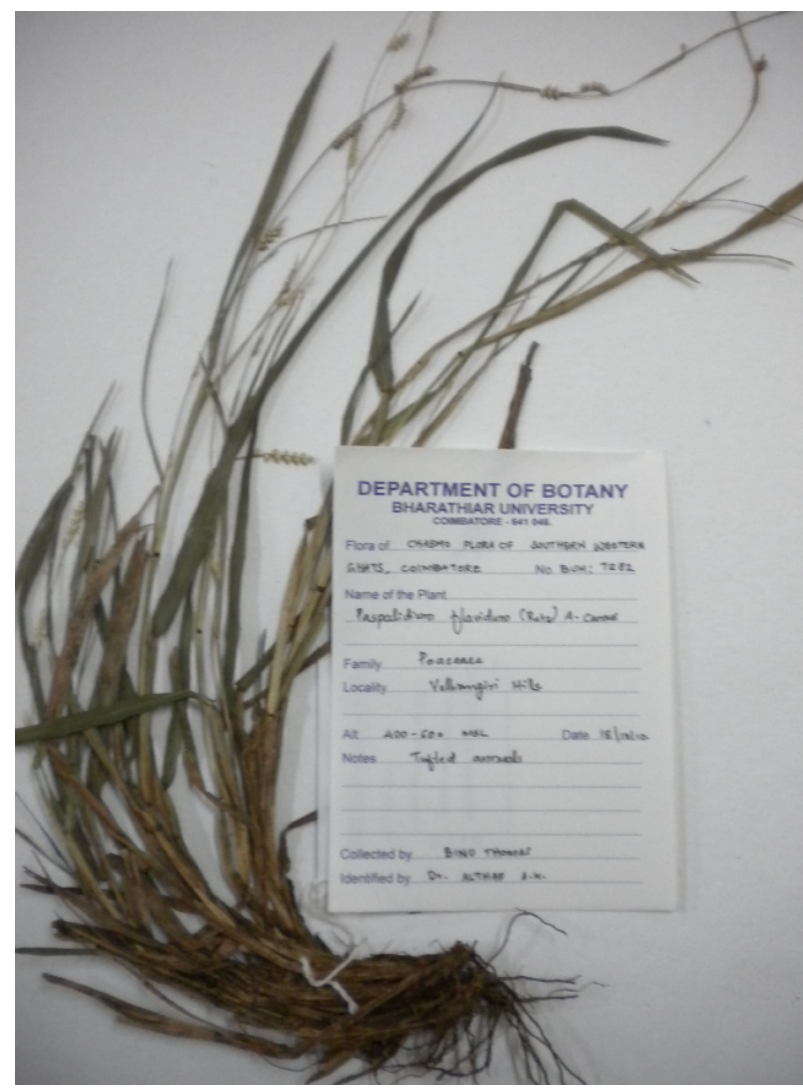

Image 26. Paspalidium flavidum (Retz.) A. Camus

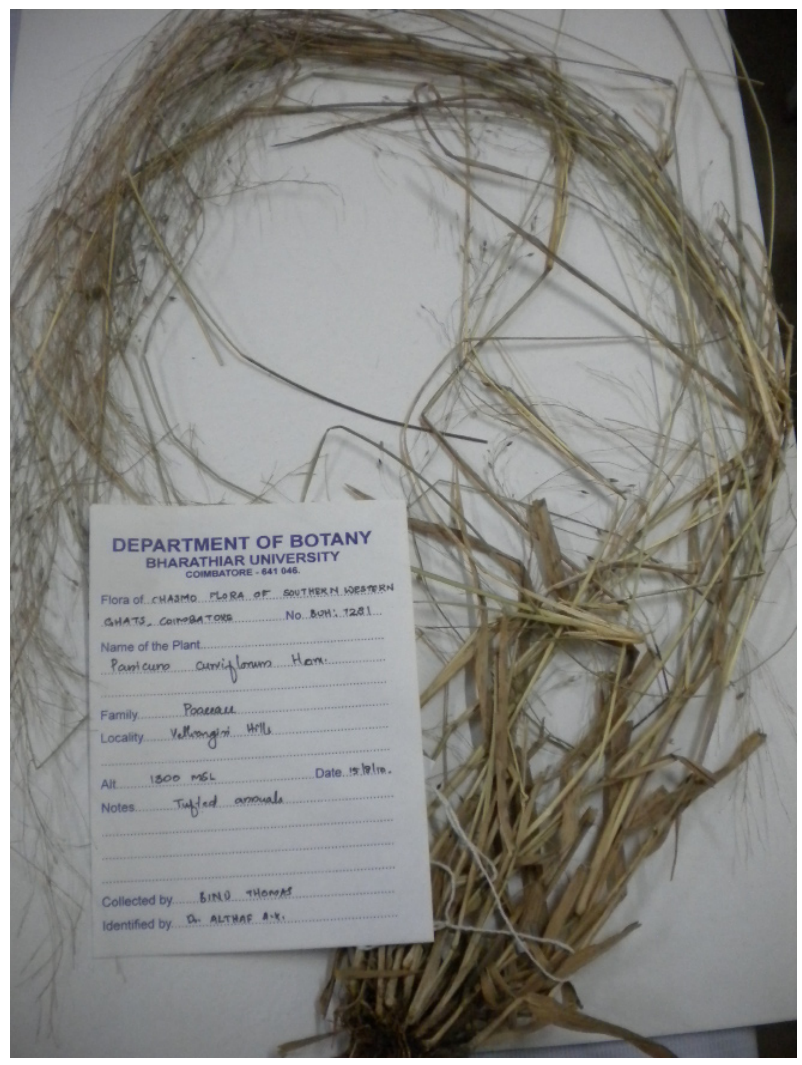

Image 25. Panicum curviflorum Hornem.

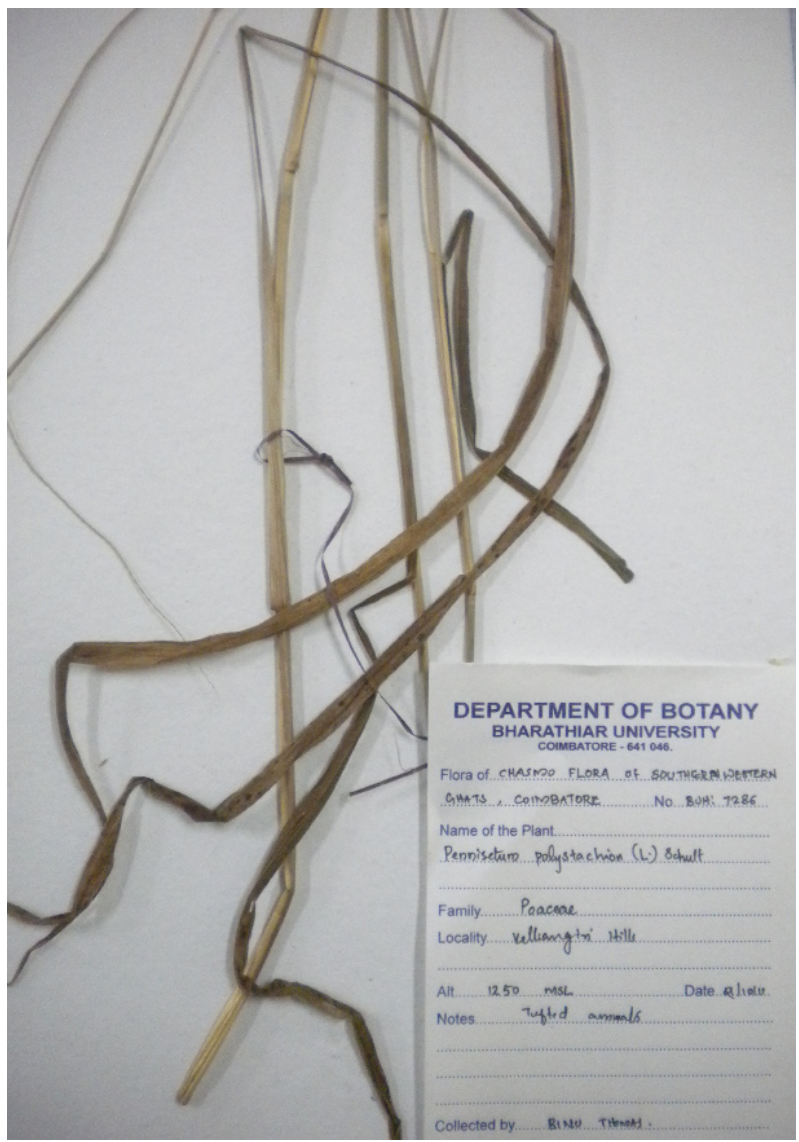

Image 27. Pennisetum polystachion (L.) Schult. 


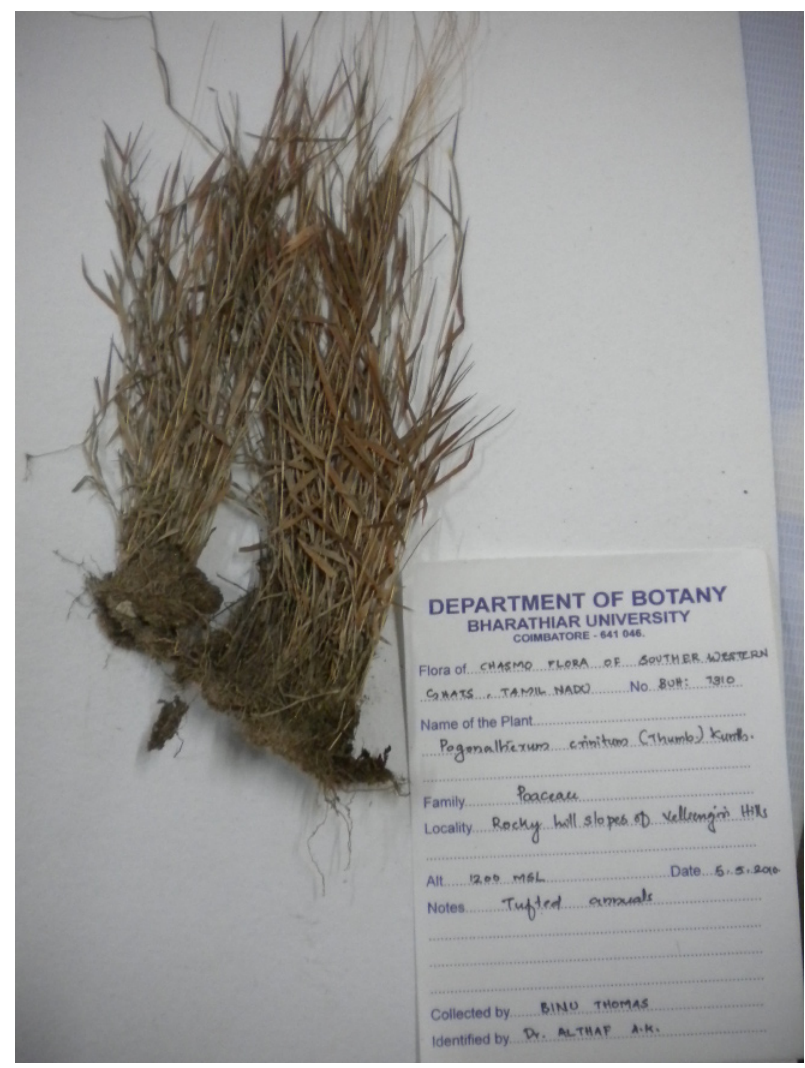

Image 28. Pogonatherum crinitum (Thunb.) Kunth.

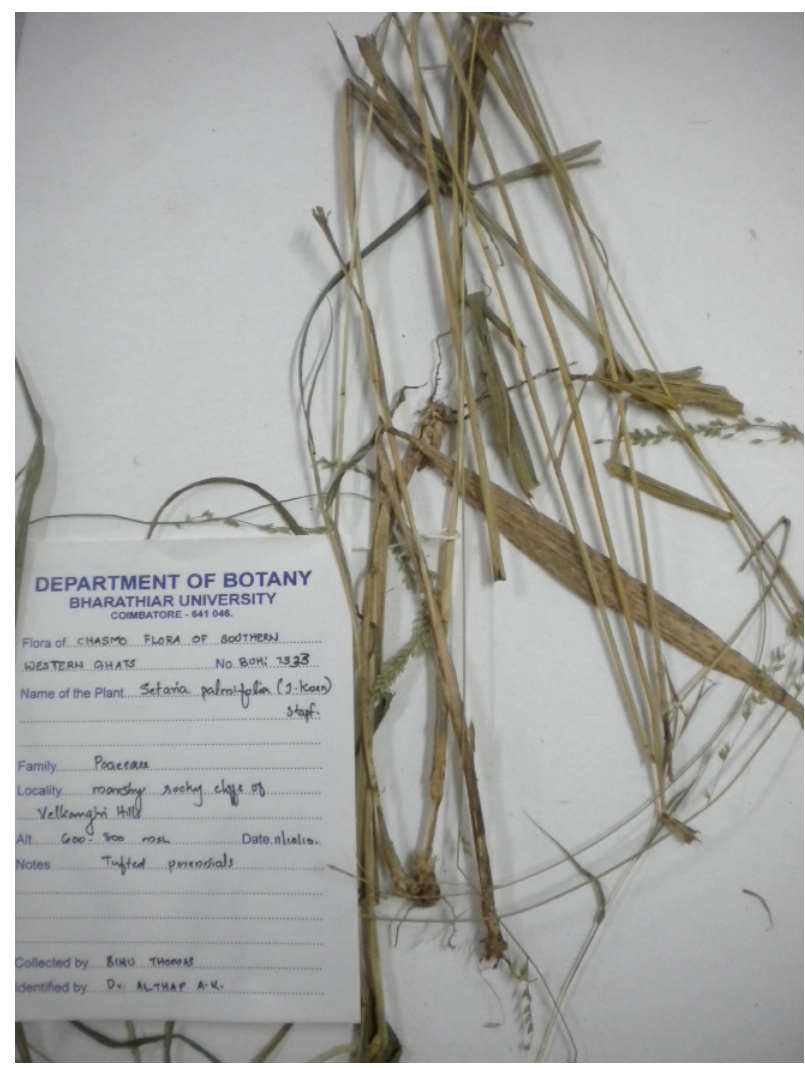

Image 30. Setaria palmifolia (J. Koenig) Stapf.

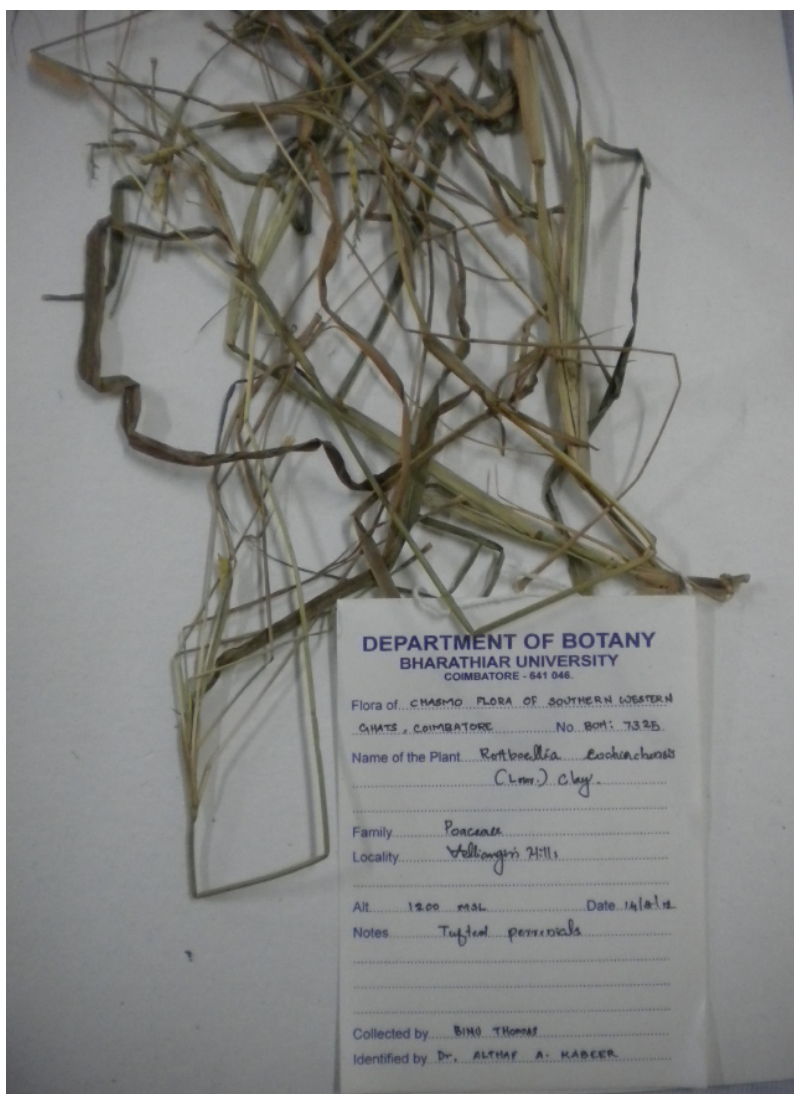

Image 29. Rottboellia cochinchinensis (Lour.) Clayton

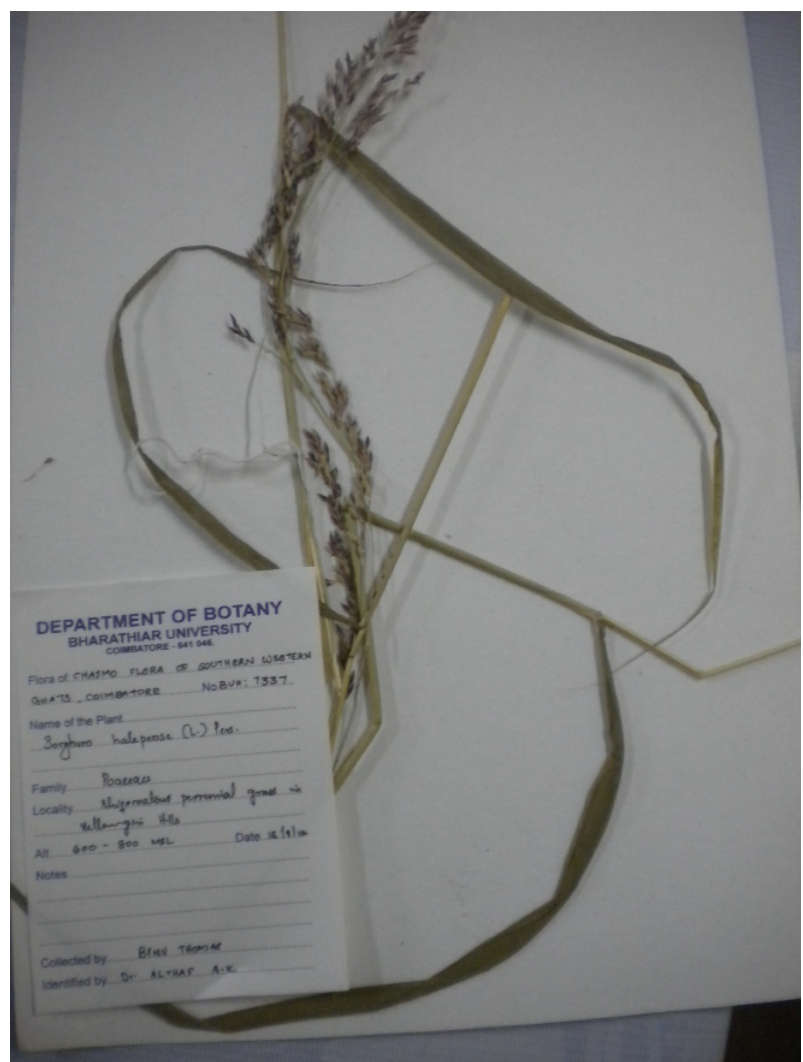

Image 31. Sorghum halepense (L.) Pers.

Journal of Threatened Taxa | www.threatenedtaxa.org | December 2012 | 4(15): 3462-3472 


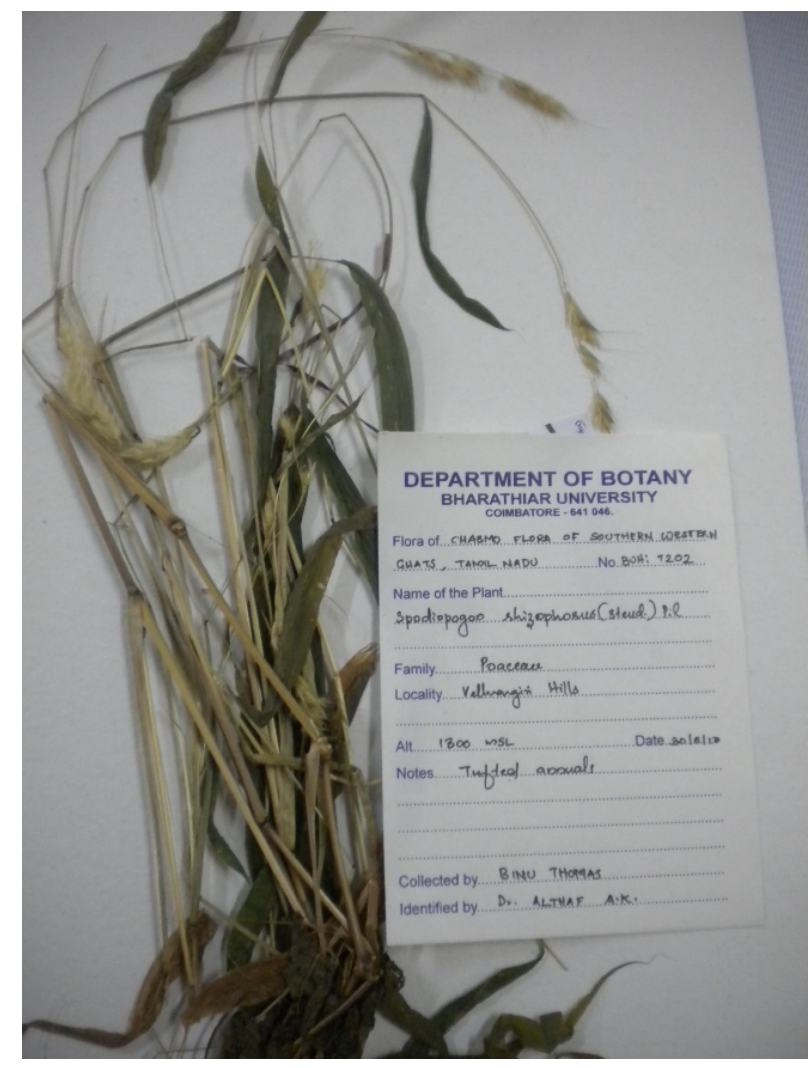

Image 32. Spodiopogon rhizophorus (Steud.) Pilger

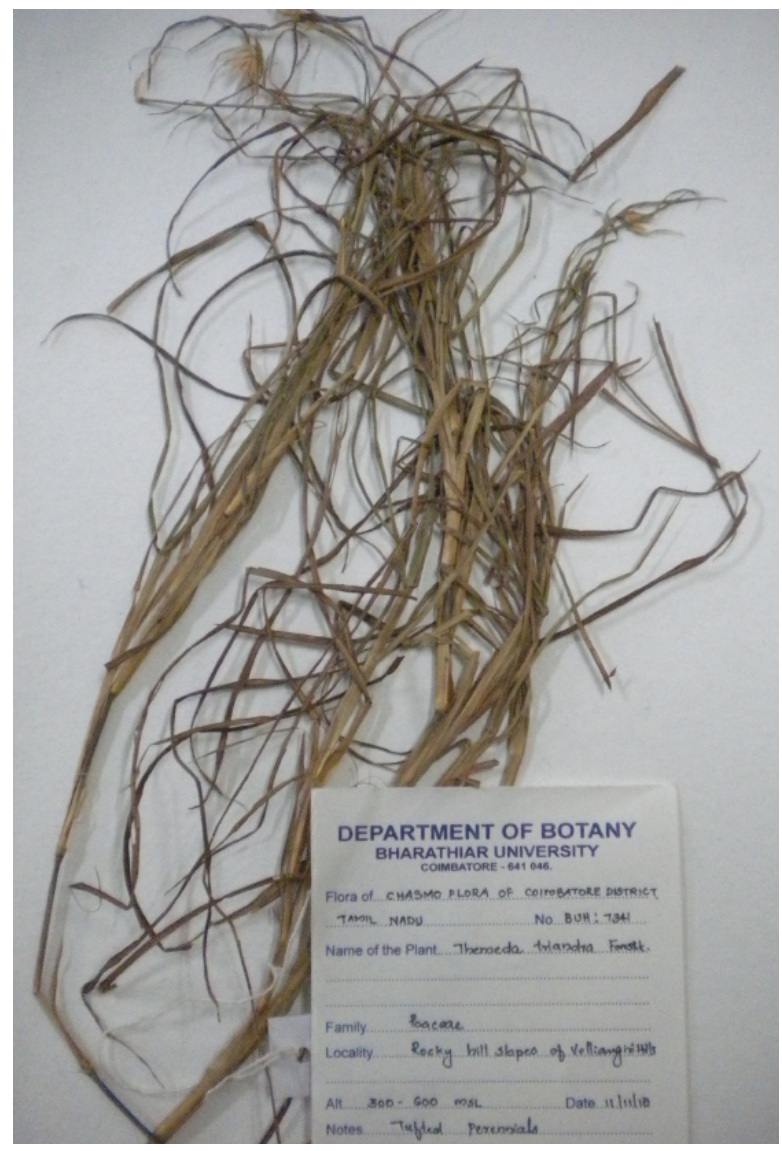

Image 34. Themeda triandra Forssk.

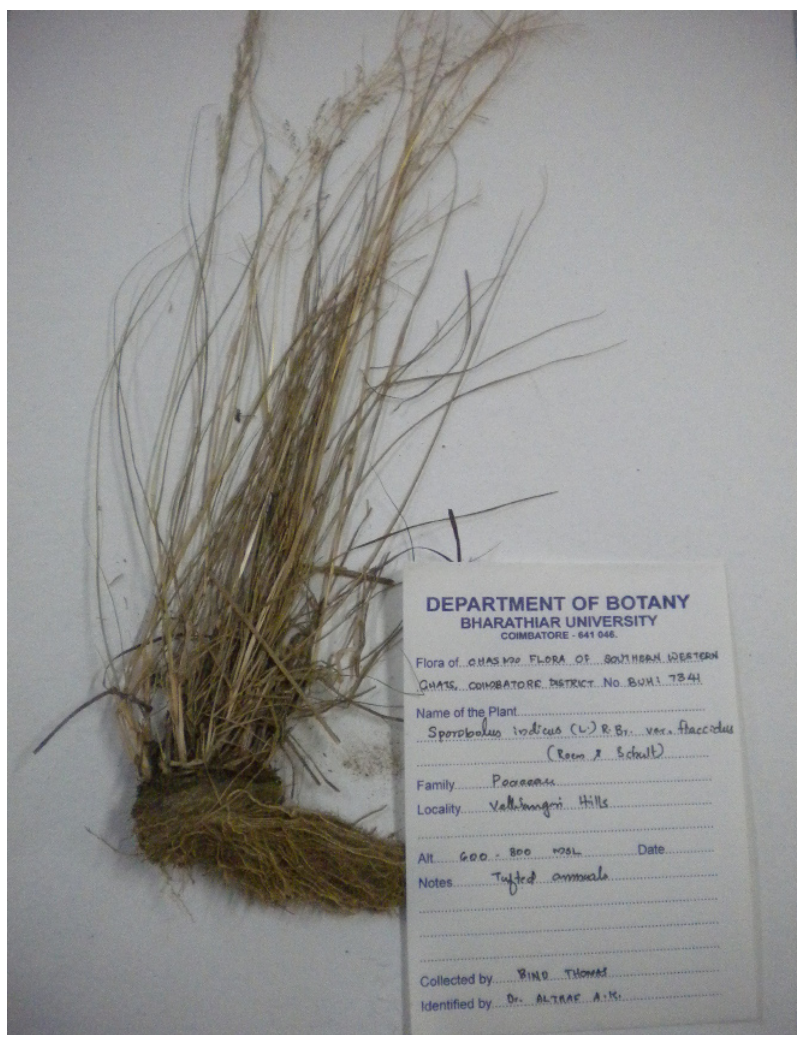

Image 33. Sporobolus indicus (L.) R. Br. var. flaccidus (Roem. \& Schult.)

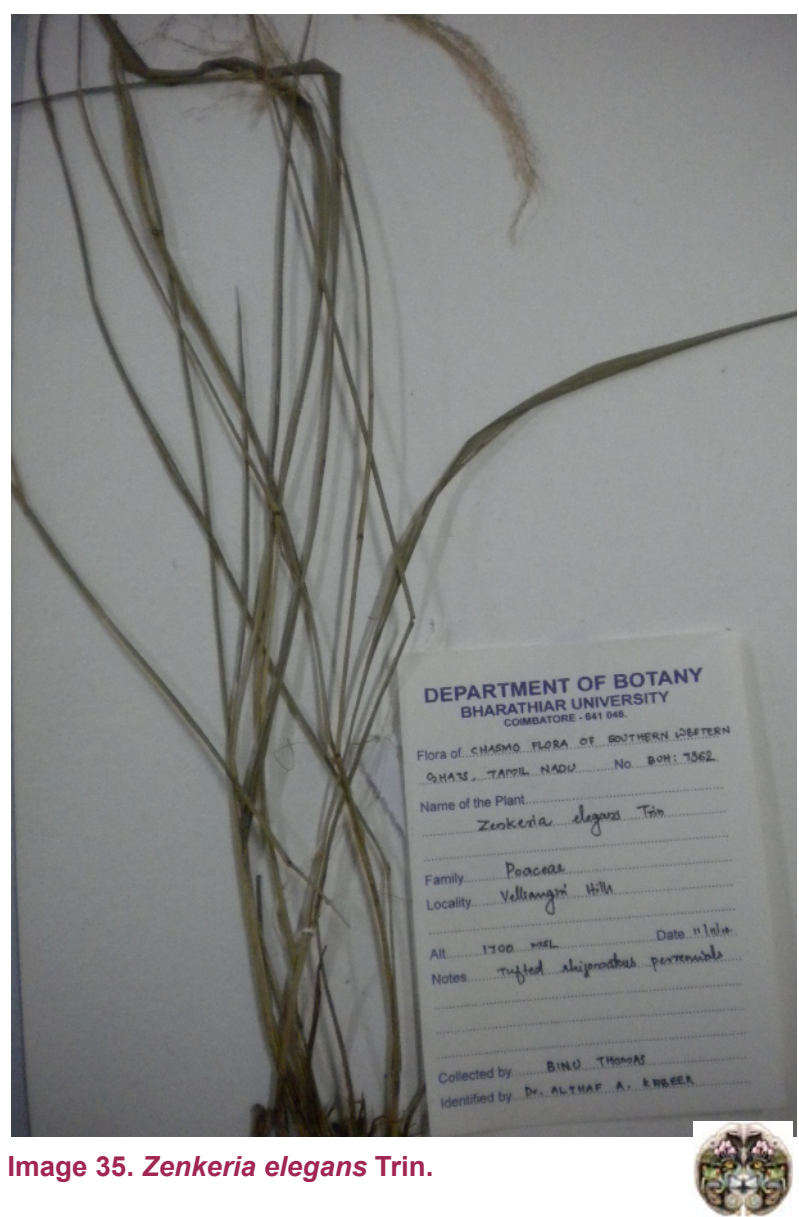

